\title{
Predicting nanoparticle uptake by biological membranes: Theory and simulation
}

\author{
M. Schneemilch, N. Quirke
}

\begin{abstract}
Department of Chemistry, Imperial College 301G Molecular Sciences Research Hub White City Campus, 80 Wood Lane, London W12 OBZ, United Kingdom
\end{abstract}

\begin{abstract}
We describe a new approach which predicts the level of internalisation or complete wrapping of nanoparticles by liposomes in solution. It is based on a generalisation of elastic theory to nanoscale particles with physical property data obtained from atomistic and coarse-grained simulations. We apply this approach to determine the maximum number of nanoparticles of a given type that can be internalised by a given liposome and give examples of how our approach might be used to identify and/or design nanoparticles with different uptakes: New data that could be correlated with nanoparticle toxicity experiments. We briefly discuss the possibility of designing nanoscale separations process.
\end{abstract}

\section{INTRODUCTION}

The interaction of inorganic surfaces with cells is of interest in medicine and in toxicology. In particular, the effects of exposure to finely divided inorganic materials in the form of nanoparticles are of great concern ${ }^{1,2,3,4,5}$ as their toxicological properties are uncertain, even for naturally occurring minerals such as silica and titania. At the same time there has been a rapid increase in the number and variety of engineered nanoparticles in the environment ${ }^{6}$, including those arising from the use of batteries, catalysts, chemical coatings and paints, packaging, electronic devices, implants, biomedicines, food additives and cosmetics ${ }^{7}$. Nanoparticles may enter the human body though inhalation, digestion, injection or through direct contact with the skin. Our research ${ }^{31,41,8}$ and that of others ${ }^{9,10}$ is focused on the interaction of nanoparticles with cytoplasmic and vesicular membranes, as a first step in assessing the likely impact of nanoparticles at the cellular level ${ }^{11}$. To help identify the essential features of such interactions we first study much a simpler 'model' membrane; the liposome ${ }^{12,13,14}$ with the long term goal of being able to predict the likely effect of nanoparticles on cells. For example, an adverse outcome ${ }^{15}$ may arise due to damage caused by nanoparticles to lysosomal membranes within the cellular endocytic (autophagy) pathway. Whilst the role of functioning lysosomes in autophagy is an essential part of cell maintenance, cell death can be triggered by lysosomal membrane permabilisation (LMP) or rupture and subsequent release of lysosomal hydrolases from the lumen into the cytosol. There is strong evidence ${ }^{16,17}$ that some nanomaterials can interfere with the functioning of lysosomes, through e.g. macrophage overload, LMP and depletion (selective adsorption) causing lysosomedependent cell death, initiating adverse outcome pathways in the body.

A key measure of the degree of interaction between nanoparticles and membranes is the extent to which the nanoparticle adheres to or, is wrapped by, the membrane. According to the Helfrich membrane model ${ }^{18}$ applied by Deserno et $a l^{19}$ to the wrapping of spherical particles by membranes, this is determined by whether the adhesion strength is sufficient to overcome the bending energy associated with membrane deformation during particle 
wrapping. Particles smaller than a critical size will not adhere to the membrane. Larger particles will adhere and undergo wrapping, the extent to which wrapping occurs is determined by the membrane tension. With low tension and/or strong adhesion, particles will be completely engulfed. However, the assumptions inherent in the model means that it is strictly only valid when the particle is small compared to the liposome diameter (small local membrane curvature) and when the particle is large compared to the membrane thickness (which is about $4 \mathrm{~nm})$.

The relevance to nanoparticle toxicity arises from three considerations a) nanoparticles which are predicted to be fully or partially wrapped may scavenge lipids from the cell plasma membrane destroying or disrupting ${ }^{20}$ it, b) nanoparticles can be taken up by the cell by endocytosis and subsequently exposed in the autolysosome where they will interact with the lysosomal membranes possibly leading to the lysosomal membrane permabilisation or rupture and subsequent release of lysosomal hydrolases, and c) fully wrapped nanoparticles, taken up by the cell by passive adsorption, could detach from the membrane inside the cell and become available to be taken up by the endocytic pathway(see b). Note that gold nanoparticles inside vesicles are observed experimentally ${ }^{21}$ to cross the plasma membrane of HeLa cells into the cytosol where a fraction are subsequently taken up by endosomes.

The adhesion strength drives the particle wrapping process and therefore any assessment of the potential nanotoxicity of a material must include an estimate of its adhesion strength for a given membrane which we approximate in what follows by the adhesion strength with respect to a model membrane featuring only lipids (ignoring for now the role of membrane proteins). A major obstacle to progress is the almost complete lack of quantitative experimental data for nanoparticle/lipid bilayer adsorption enthalpies and free energies ( adsorption strength) ${ }^{22}$. One solution is to calculate such data using molecular simulation and indeed in previous work we have calculated adhesion strengths for DMPC bilayers on planar surfaces of gold ${ }^{23}$, silica ${ }^{24}$ , and titania ${ }^{25}$.In what follows we describe a mechanistic model of NP uptake by model membranes which, given several assumptions, can be used to rank NPs with respect to their ability to disrupt and/or penetrate lipid membranes. We use molecular simulation and elastic theory to treat the problem of nanoparticle wrapping by liposomes which, in principle, enables a wide range of nanomaterials to be screened with the minimum of time-consuming simulations. This has enabled us to form a ranking of nanomaterial surfaces based on lipid membrane adhesion strength (table 1) from simulation for a subset of materials used in the SmartNanoTox ${ }^{9}$ project. Adhesion strength data could be used to develop models/rankings of potential cell damage due to a given type of nanoparticle and/or pulmonary surfactant disruption due to the presence of nanoparticles in the alveolar spaces of lungs. An important finding is that there are no shortcuts to the prediction of adhesion strength. For example, estimating the adhesion strength based on single molecule adsorption data does not give the right answer because the physics of the two processes is different. Membrane lipids orient perpendicular (or at an acute angle with respect to the surface normal) to the surface while single or low density lipids tend to adsorb flat on the surface.

Our work can be divided into two broad sections. In the first, we estimate the strength of adhesion by conducting atomistic scale classical molecular dynamics (MD) of lipids on gold, silica, and titania surfaces. In the second section, details of coarse grained (CG) simulations of cylinders, spheres and cubes with sizes comparable to the bilayer thickness are discussed and used to determine appropriate modifications to the thin film model so that it may be applied to membranes of finite thickness wrapping spherical and cylindrical particles. 


\section{METHODS}

Details of the simulation geometries and force fields used to calculate the adhesion strengths are briefly described. The following section introduces the coarse grain model and simulations conducted to characterise its bilayer properties. The thin film theory is described and the extension of the theory to accommodate membranes of finite thickness discussed. The research described in the next two sections are encoded in the programme Nanolip (see section 3.3)

\subsection{ADHESION STRENGTH}

The change in free energy per unit area upon the adhesion of a bilayer from solution onto a surface, is here referred to as the adhesion strength. It may also be considered as the force per unit length required to prevent a semi-adsorbed bilayer ribbon (as depicted in Figure 1) from further adhesion. The constraint force, $F$, required to keep the end of the ribbon at a distance $h$ above the surface is monitored during the simulation. The constraint force is related to the adhesion strength $k_{w}$ by ${ }^{23}$

$$
k_{w}=F \frac{d h}{d A}
$$

where $d A$ is the increase in the area of the adsorbed bilayer resulting from a reduction in the restraint height by $-d h$. The adhesion strength, as a change in free energy, is negative when adhesion is spontaneous. The ratio $d h / d A$ is the product of twice the inverse of the area per lipid in the supported lipid bilayer, and the inverse of $d n / d h$, where $\mathrm{n}$ is the number of lipids in the free section of the bilayer. The ratio $d n / d h$ is estimated from the one-dimensional lipid number density profile perpendicular to the surface at the point where the magnitude of the gradient is at the minimum. Note that the adhesion strength can be expected to be independent of the restraint height as long as the length of the ribbon is sufficient to ensure that at least some portion of the adhering ribbon is representative of the 'bulk' adhesion density i.e. is not affected by proximity to the edges.
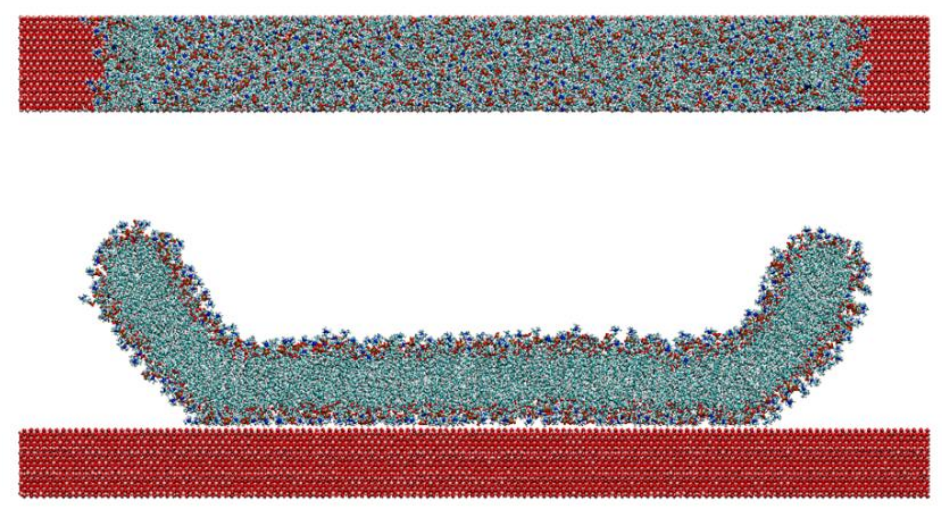

Figure 1. Top and side views of a lipid ribbon adsorbed on the rutile 100 surface. Water is omitted for clarity.

The DMPC lipids comprising the bilayer ribbons were modelled using the fully atomistic 118 site Slipids forcefield which was developed ${ }^{26,27,28}$ for use in conjunction with TIP3P water. This system was shown to reproduce many experimentally observed properties of bilayers such as the lipid specific area and volume, bilayer thickness, isothermal area compressibility and NMR order parameters and scattering form factors.

Surface slabs were constructed with surface dimensions of approximately 30 by $4 \mathrm{~nm}$. Equilibrated bilayer ribbons were then brought into contact with the slab surface. The edges 
of the ribbon were maintained at distance of $8 \mathrm{~nm}$ from the surface using the GROMACS pull code with a harmonic constraint with force constant of $10 \mathrm{~kJ}^{-m_{0}}{ }^{-1}$. A total of 18 lipids comprised the pull group at each edge of the ribbon. The distribution of lipids in the ribbon was maintained at equilibrium by periodically updating the lipids in the pull group at $0.5 \mathrm{~ns}$ intervals. The simulation was halted, and lipids were reassigned to the pull groups based on the position of the head groups. Those lipids with phosphorous sites furthest from the solid were assigned to the pull groups, allowing individual lipids to migrate around the ribbon edge and maintain equal tension in both leaflets.

We have used this method to calculate adhesion strengths for DMPC bilayers on planar surfaces of gold $^{29}$, silica ${ }^{30}$, and titania ${ }^{31}$ ( see table 1) as well as mixed lipid membranes with and without cholesterol for gold.

\subsection{COARSE GRAIN MODEL}

DMPC lipids were modelled using a 10-bead implicit solvent model optimised using MAGIC software $^{32}$ to reproduce radial distribution functions generated from atomistic classical MD bilayer simulations. A schematic diagram of the model is shown in Figure 2. Note that there are 4 different types of bead, $\mathrm{C} 1$ and $\mathrm{C} 5$ being of the same type.

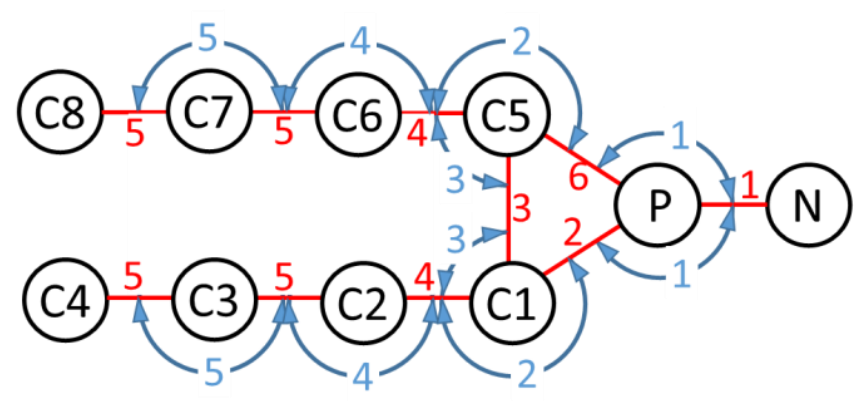

Figure 2. Schematic of the CG DMPC lipid. Beads are shown as black circles, bonds are shown as red lines and blue arrows indicate angles.

The CG bilayer elastic modulus was determined from a series of fully periodic simulations where the number of lipids was fixed and the area per lipid (ApL) was varied by scaling the lateral dimensions of the simulation cell. The tension in the bilayer was measured in each simulation and plotted in for a range of different tensions.

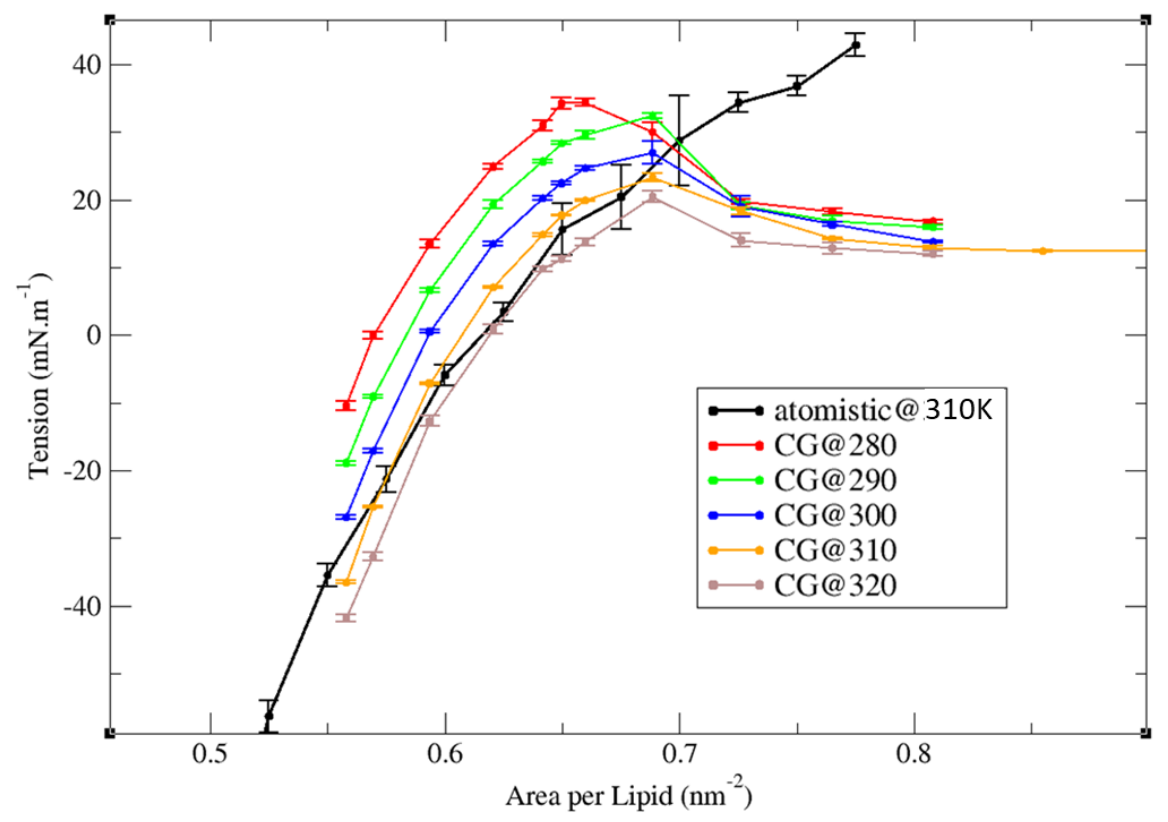

Figure 3. Membrane tension as a function of ApL for the CG bilayer over a range of temperatures. The black line indicates the behaviour of the atomistic bilayer. 
In figure 3 the elastic behaviour of the atomistic model is shown in black. The elastic modulus is determined by the slope of the curve at zero tension and the rupture tension is determined from the maximum tension. For the atomistic module the rupture tension is approximately 45 $\mathrm{mN} . \mathrm{m}^{-1}$, but rupture tensions for the CG model are lower - especially for higher temperatures. However, at lower temperatures the ApL at zero tension is significantly less than that of the atomistic model. Therefore we chose to simulate the $\mathrm{CG}$ bilayer at $300 \mathrm{~K}$ where the rupture tension is approximately $25 \mathrm{mN} \cdot \mathrm{m}^{-1}$.

The CG bilayer bending modulus was estimated by actively deforming the lipid bilayer and measuring the curvature and restorative forces ${ }^{33}$. A schematic of the simulation cell is shown in figure 4.

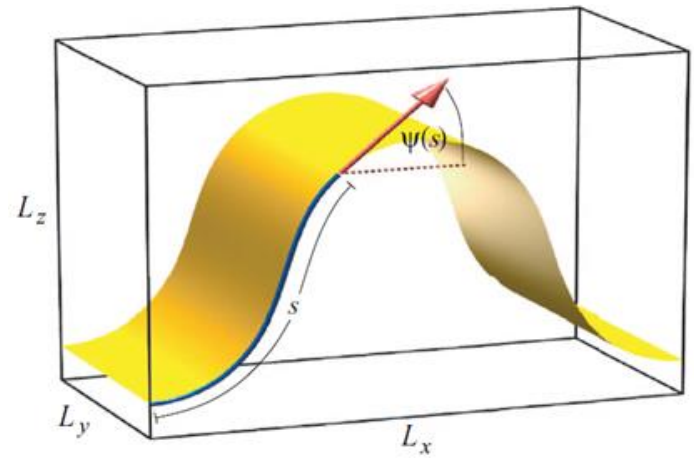

Figure 4. An illustration of the buckled membrane, where $L_{x}, L_{y}$ and $L_{z}$ are the dimensions of the box, $s$ is the coordinate measured along the membrane, and $\Psi(s)$ is the angle between the tangent to the membrane and the $\mathrm{x}$-axis.

The equation connecting the restorative force in the $x$-direction, $F_{x}$, the bending strain, $\gamma$, and the bending modulus, $\kappa$, is given by:

$$
F_{x}(\gamma)=4 \pi^{2} \kappa \frac{L_{y}}{L^{2}} \sum_{i=0}^{\infty} b_{i} \gamma^{i},
$$

Where $L_{y}$ is the is length of the simulation cell in the $y$-direction, $L$ is the length of the membrane and $b_{i}$ are the series coefficients given in reference 33. The bending strain, $\gamma$, is defined as the proportion by which the projected length of the membrane is reduced by the buckling:

$$
\gamma=\frac{L-L_{x}}{L}
$$




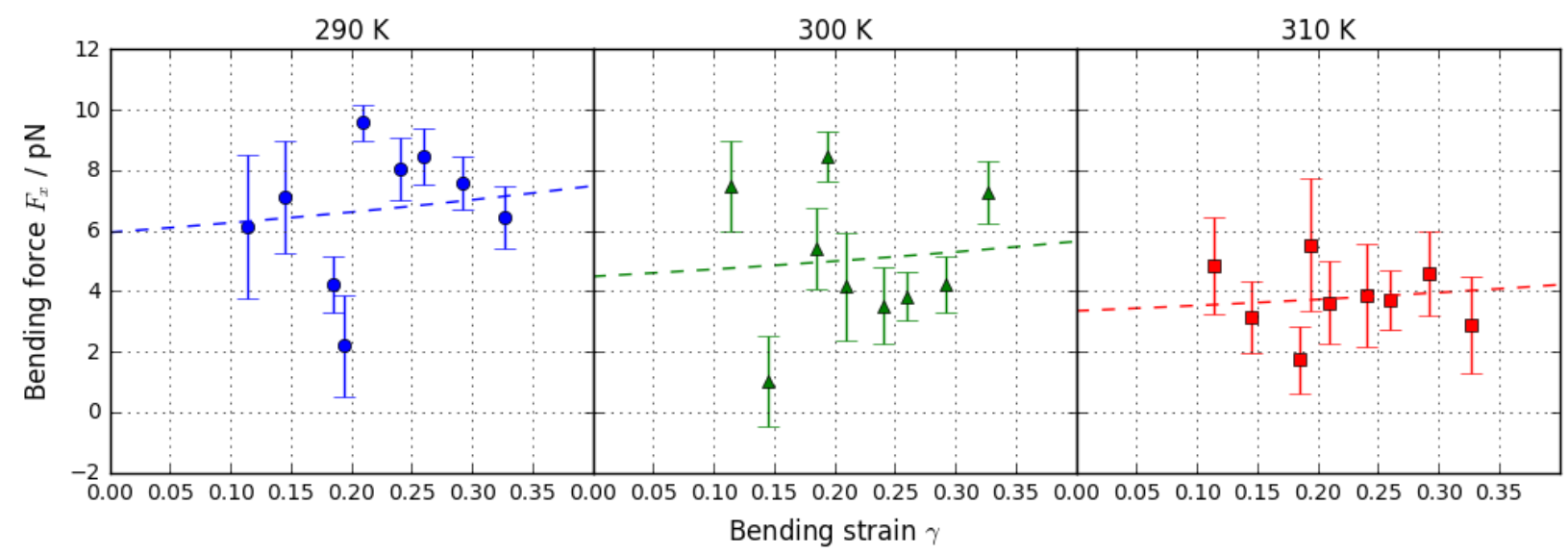

Figure 5. Plots of restorative bending forces in the $\mathrm{x}$-direction against bending strain with fits to equation 2 shown by the dashed line.

Plots of $F_{x}$ against $\gamma$ are shown in the figure 5 for a range of temperatures along with fits to equation 2.The general trend of $F_{x}$ is to slightly increase with greater strain, which follows from the definition of $F_{x}$ in Equation 2. , the slope of the fit does not significantly change with $\kappa$, but the $y$-intercept does. It can be explained by expanding equation 2 when $i=0, \gamma$ vanishes and leaves a constant term containing $\kappa$. A change in $\kappa$ can proportionally affect the constant term, which equals the $y$-intercept, but the terms with higher power of $\gamma$ is insignificant as $\gamma \ll 1$, so the slope does not change much. Consequently, we can directly estimate the bending modulus by inspecting the y-intercept.

As might be expected intuitively, the bending modulus decreases with temperature. At $300 \mathrm{~K}$ the CG bending modulus is $3.6 \pm 0.6 \times 10^{-20} \mathrm{~J}$. Although significantly less than the published value of $11 \times 10^{-20} \mathrm{~J}$ for the bending modulus determined from atomistic simulations, it is of the same order of magnitude and therefore suitable for our purposes.

Next, potential of mean force (PMF) simulations were conducted for atomistic models of each of the four types of CG bead interacting with a gold slab in vacuum. The PMF curves were fitted with the 10-4-3 Steele potential to obtain the effective $\sigma$ value of the LJ potential for each bead when interacting with the substrate atoms. These values were then used in conjunction with the LJ well depth, $\epsilon$, generated from Lorenz-Bertholet mixing rules using the gold-gold and bead-bead well depth values to generate a PMF curve for a CG lipid interacting with the substrate. This was repeated; each time scaling the $\epsilon$ values by a different factor. The results are shown below. 


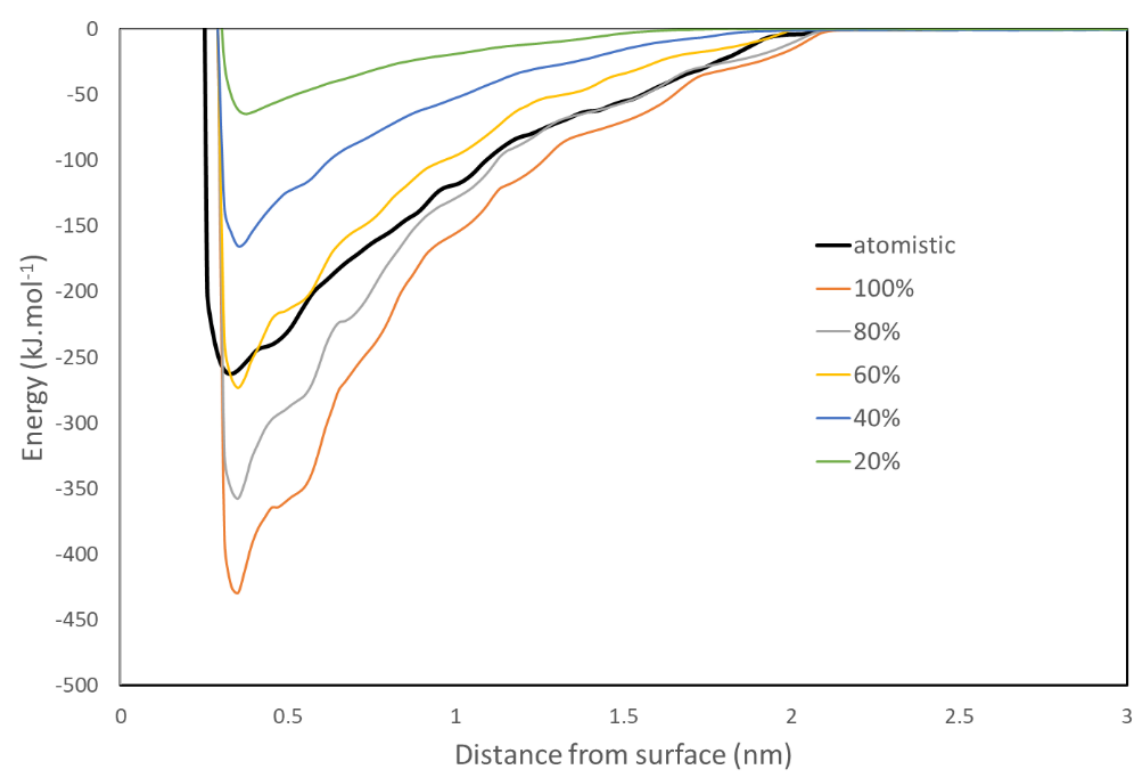

Figure 6. PMF curves for a CG lipid interacting with an atomistic slab. The black line denoted the PMF for an atomistic lipid and a gold slab in water.

When the $\epsilon$ values are scaled by $60 \%$ the implicit solvent CG model approximates well the explicit solvent atomistic PMF. Next adhesion strength measurements were performed against the slab with a CG bilayer using the ribbon geometry, varying both the scaling factor and the temperature. The results are shown below.

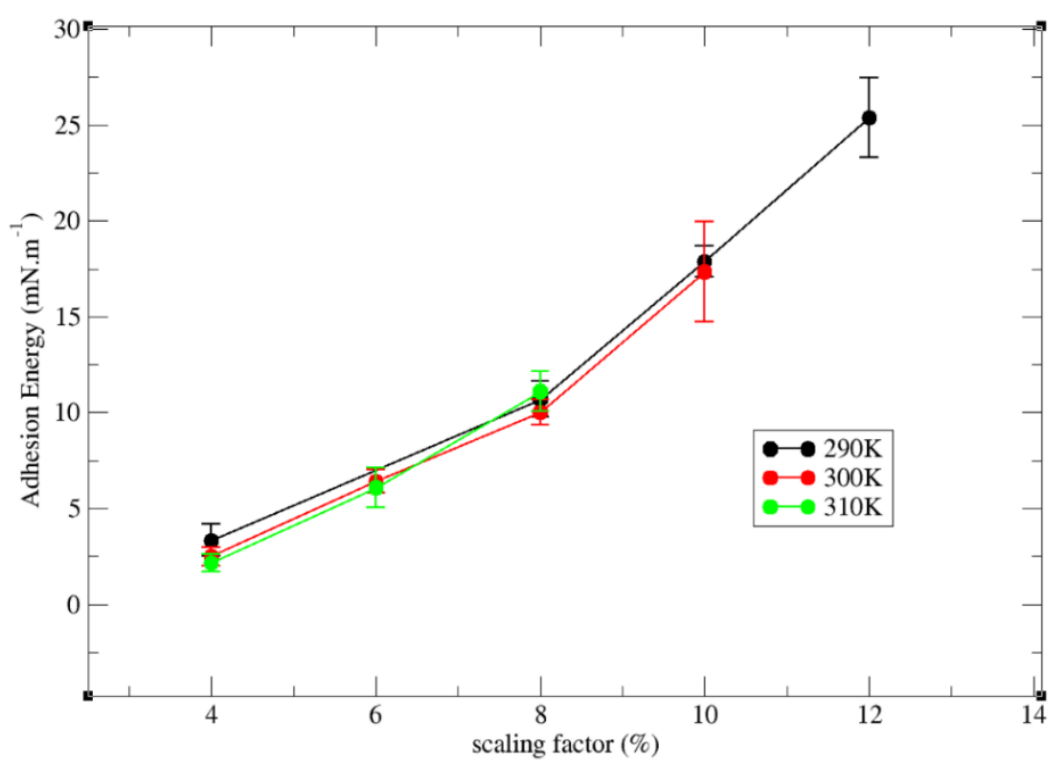

Figure 7. The adhesion strength of $C G$ bilayers on the slab using a range of scaling factors and temperatures.

The upper limit on the adhesion strength measurable for a given temperature is set by the rupture tension identified in Figure 3. Within these limits, any desired adhesion strength can be selected by choosing the appropriate scaling factor. The gold lattice forming the substrate can be used to model particles of any shape and size. 


\subsection{WRAPPING OF A SPHERE}

Simulations of spherical particle wrapping were conducted using CG lipid bilayers in a disk configuration. In a 2-dimensional analogue of the 1-dimensional ribbon, tension is maintained in the disk by identifying a series of pull groups around the disk perimeter, each consisting of

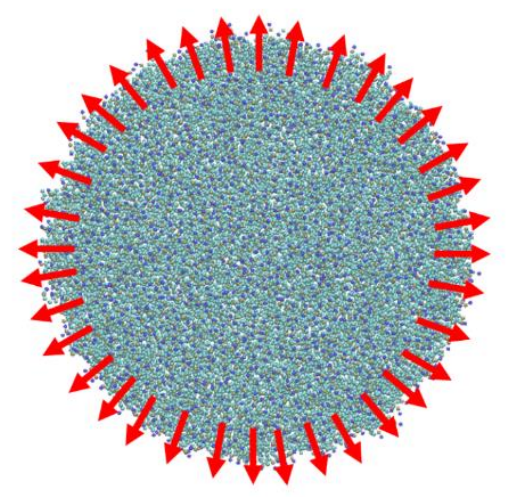
approximately 18 lipids. Unidirectional restraining potentials are applied to maintain a minimum distance between the pull groups and the centre of the disk. The simulation geometry is illustrated in Figure 8.

Figure 8. Snapshot of a CG bilayer in the disk configuration. Red arrows show the force acting on the pull groups. The disk contains $2400 \mathrm{CG}$ lipids and the disk is $30 \mathrm{~nm}$ in diameter.

The instantaneous tension in the disk can be easily determined by the sum of the restraining forces acting on each peripheral pull group divided by the perimeter of the disk. The dependence of the disk tension on the ApL was determined by varying the restraint distance between the centre and the pull groups. Figure 9 shows this dependence, depicted by orange points, along with the dependence measured in a fully periodic simulation cell. At greater tensions the agreement is close, however there is a divergence as the tension vanishes. The reason for the difference is that when the apl gets close to zero the infinite plane system experiences negative tension or compression as the apl fluctuates which serves to give a lower tension at a given apl, whereas the disk does not go into compression. Since the disk is the geometry in the wrapping simulations this is the appropriate dependence to use for our analysis. In the case of a liposome, it will presumably not go into compression either, as it can rather deform to a non spherical shape. In principle, if the planar simulation cell was large enough it could deform as well, so compression can be viewed as an artefact of the cell size.

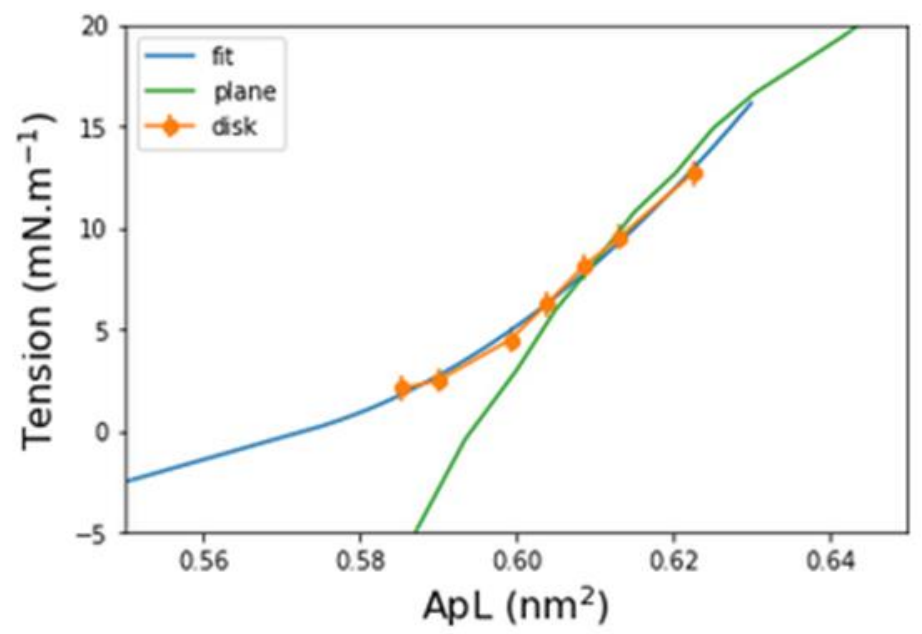

Figure 9. The dependence of the membrane tension on ApL. The tension measured in the disk geometry is shown in orange and the fit illustrated by the blue line. The green line is the dependence generated using a fully periodic bilayer.

Spheres were cut from a gold lattice and placed at the centre of the disk. Harmonic constraints maintained the particle on the central axis running perpendicular to the disk during the wrapping process. In addition to the restraints on the peripheral pull groups maintaining the lateral distance from the central axis, additional constraints acting along the direction of the central axis maintained the pull groups at a fixed reference position to ensure the edge of the disk remained flat. 

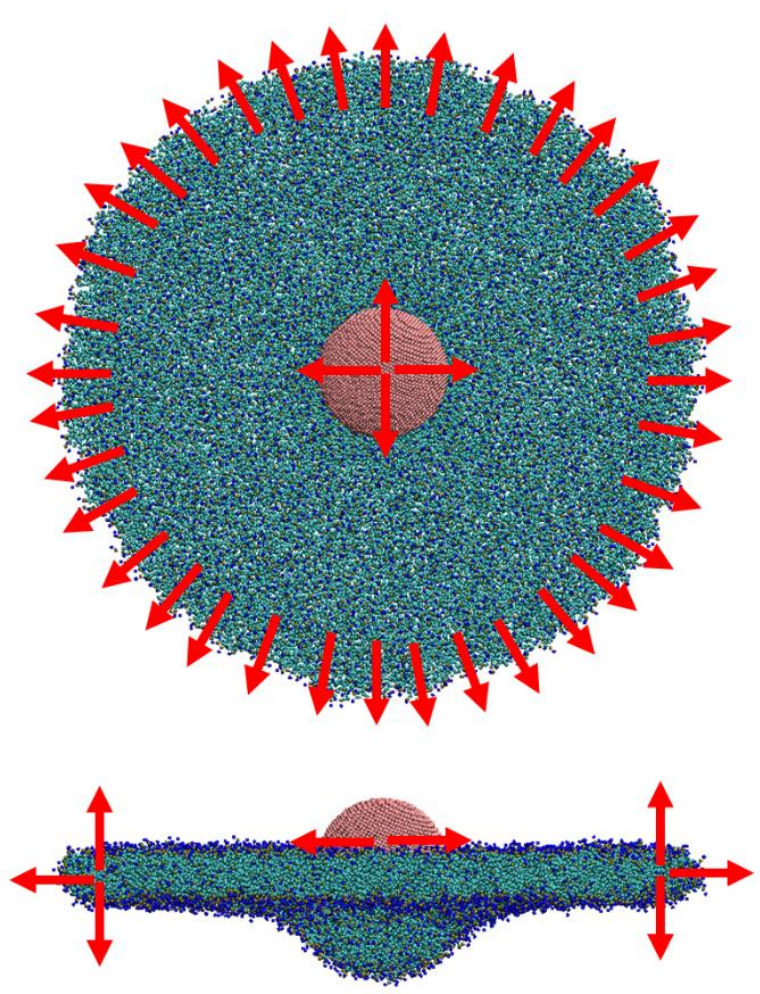

Figure 10. Top and side views of a $10 \mathrm{~nm}$ particle undergoing wrapping by a CG bilayer in the disk geometry. Red arrows show the direction of restraining potentials. Note that not all the peripheral position restraints are shown in the side view.

\subsection{WRAPPING OF A CYLINDER}

Simulations of cylindrical particle wrapping were conducted using the ribbon configuration, so that in effect, the cylinders were infinite in length. Snapshots of the configuration are shown in Figure 11 including the direction of action of the position restraints. The width of the simulation cell in the direction of the cylinder axis was $10 \mathrm{~nm}$, and three restraints were used to maintain tension at each end of the ribbon, maintaining a minimum distance between the edge and the cylinder axis. In addition, the edges were restrained to maintain a constant vertical position and a further two restraints were added $5 \mathrm{~nm}$ from the edge to ensure the edges remained flat. Finally, a restraint maintained the cylinder in the centre of the ribbon.
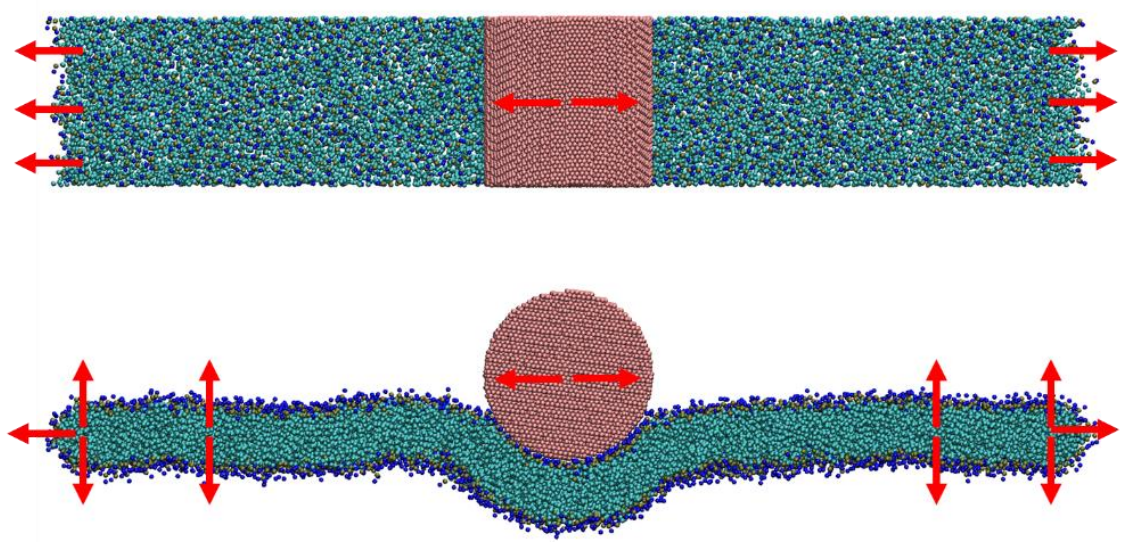

Figure 11. Top and side views of a $10 \mathrm{~nm}$ cylinder undergoing wrapping by a CG bilayer in the ribbon geometry. Red arrows show the direction of restraining potentials.

\subsection{THIN FILM THEORY}


A theoretical description of a spherical particle adhering to a flat, infinitesimally thin elastic membrane was provided by Deserno ${ }^{19}$. Figure 12 , taken from his paper, depicts the situation.



Figure 12. Illustration of the wrapping geometry and membrane parametrization. An asymptotically flat membrane adheres partially to a spherical colloid of radius a with a degree of wrapping given by $z=1-\cos \alpha$. Cylindrical symmetry around the $e_{h}$ axis is assumed.

Deserno considers wrapping under constant membrane tension. In our simulation geometry, the distance $R$ is fixed. It follows that the total area of the membrane, $A$, is given by:

$$
A=2 \pi a^{2} z+2 \pi \int r(s) d s
$$

where $a$ is the particle radius, $r$ is the radial distance and $s$ is the arc length. The first term in equation 4 represents the area in contact with the particle and the second term represents the free membrane

The tension $\sigma$ and bending modulus $\kappa$ can be used to define a characteristic membrane length $\lambda$

$$
\lambda:=\sqrt{\frac{\kappa}{\sigma}}
$$

When the length scale is smaller than $\lambda$ the bending energy dominates the energy balance while at larger length scales tension dominates. A particle will only adhere to a membrane when the contact energy $w$ is sufficient to meet the energy cost of bending the membrane around the surface of the particle. This suggests we define a new shape dependent term that combines the contact energy and bending cost, that for a sphere is written as

$$
w^{s}=w-\frac{2 \kappa}{a^{2}}
$$

Since a particle will adhere to a membrane when $w_{s}>0$, we can define a critical radius, $r_{\text {crit }}$ which denotes the minimum particle size for which adhesion will occur. 
For a given value of $z$, the total energy of the system is given by:

$$
E(z)=-2 \pi a^{2} w^{s} z+\int_{A(0)}^{A(z)} \sigma(z) d A+\pi \kappa \int_{0}^{r(s)=R} r\left(\dot{\psi}+\frac{\sin \psi}{r}\right)^{2} d s
$$

Here, the first term represents the adhesion energy less the bending energy associated with the adhering portion of the membrane, the second term the tension energy associated with the increase in area due to membrane deformation and the third the bending energy in the free part of the membrane.

For a given value of $R$, the equilibrium degree of wrapping occurs when:

$$
\frac{d E(z)}{d z}=0
$$

For each value of $z$, the tension and profile shape can be determined by minimising

$$
E_{\text {free }}=\pi \int_{0}^{r(s)=R} r\left\{\kappa\left(\dot{\psi}+\frac{\sin \psi}{r}\right)^{2}+2 \sigma(1-\cos \psi)\right\}+\lambda_{r}(\dot{r}-\cos \psi)+\lambda_{h}(\dot{h}-\sin \psi) d s
$$

subject to the requirement that:

$$
\sigma=\sigma_{f i t}\left(\frac{2 A}{N_{l i p}}\right)
$$

where the dependence of the membrane tension on the ApL has been determined from simulation. The data used to determine this dependence are displayed in Figure 9. Numerical solution of these equations provides a theoretical description of the relation between $z$ and $\sigma$.

In the case of an infinitesimally thin membrane wrapping a cylinder we can write the following equation for $w^{c}$ which combines the contact energy and bending energy for wrapping of a cylinder:

$$
w^{c}=w-\frac{\kappa}{2 a^{2}}
$$

The energy equation for the cylinder wrapping by an asymptotically flat membrane is

$$
E(z)=-w_{c} 2 \alpha a L+\int_{A(0)}^{A(z)} \sigma(z) d A+\kappa L \int \dot{\psi}^{2} d s .
$$

The profile can be calculated for a given value of $\alpha$ from

$$
\psi(s)=4 \tan ^{-1}\left[\tan \left(\frac{\alpha}{4}\right) e^{-\frac{s}{\lambda}}\right] .
$$


With the curvature given by

$$
\dot{\psi}(s)=\frac{4 e^{s / \lambda} \tan \frac{\alpha}{4}}{\lambda\left(e^{2 s / \lambda}+\tan ^{2} \frac{\alpha}{4}\right)}
$$

\subsection{EXTENSION OF THIN FILM THEORY TO BILAYERS}

Thin film theory is extended to bilayer wrapping by assuming the that the bilayer behaves the same as a thin film acting through the bilayer midplane. Since the thickness of a free bilayer is approximately $4 \mathrm{~nm}$, this means that the wrapping radius, $a$, is equal to the nominal radius plus $2 \mathrm{~nm}$. In addition, is it necessary to adjust the contact energy, $w$, since it is now dependent on the particle curvature. So we apply the following definitions:

and

$$
w_{S L B}^{S}=-k_{w}+\frac{p_{1}}{a}+\frac{2 p_{2}}{a^{2}}
$$

$$
w_{S L B}^{c}=-k_{w}+\frac{p_{1}}{2 a}+\frac{p_{2}}{2 a^{2}}
$$

where the subscript SLB denotes a supported lipid bilayer and its application to the bending constant recognises that it may differ from that of a free bilayer. The contact energy $w$ given in equations 6 and 11 has been replaced by a pair of second order polynomial functions of the effective wrapping radius, $a$, where the intercept at infinite curvature is the flat surface adhesion strength measured in simulation, $-\mathrm{kw}$. The parameters $\mathrm{p} 1$ and $\mathrm{p} 2$ will be determined empirically from coarse grained simulations of particle wrapping.

In the cylindrical geometry $w_{S L B}^{c}$ can be measured directly by applying restraints to maintain the ends of the ribbon a minimum distance from the centre of the cylinder. In this case there is no bending energy in the free part of the bilayer and so the tension in the membrane is equal to the effective adhesion strength. Therefore, simply dividing the restraining force by the width of the ribbon yields $w_{S L B}^{c}$. The simulation geometry is illustrated below.



Figure 13. Snapshot showing the simulation geometry for determination of $w_{S L B}^{c}$ in the case of cylinder wrapping 
In the spherical geometry, $w_{S L B}^{S}$ cannot be determined directly in this manner, so must be estimated by fitting the thin film model to the wrapping data. We have chosen to fit the dependence of the membrane tension on the disk radius $R$. but could have also used the penetration depth.

\section{RESULTS}

Adhesion strength simulations and coarse grain results are presented below.

\subsection{ADHESION STRENGTH}

The results of the adhesion simulations are recorded in Table 1, data taken from previously published work for DMPC on gold ${ }^{29}$, silica ${ }^{30}$, and titania ${ }^{31}$.By far the greatest adhesion strength was recorded on the gold surface. For this material a reliable experimental value has been determined from chronocoulometry measurements with a gold electrode ${ }^{34}$. Integration of the difference between charge density curves in the presence and the absence of an adhering bilayer, respectively, yields a value of $-40 \mathrm{mN} \cdot \mathrm{m}^{-1}$ for $k_{w}$. The value reported in the table was achieved by calibrating the gold-lipid interaction; scaling by a factor of 0.95 the LJ well depth of each individual pair potential. The adhesion enthalpy per unit area, $\Delta H_{a d h}$, here was estimated from the system enthalpy measured with one of the ribbon edges restrained at a range of distances from the surface, effectively a direct measurement of the enthalpy change with respect to adhesion area. The entropic contribution to adhesion per unit area, $T \Delta S$, can be inferred from $w$ and $\Delta H_{a d h}$ via the Gibbs free energy equation. In the case of gold adhesion, the entropic contribution opposes adhesion, which may be intuitively expected on the basis of the reduction of freedom of movement of the adhering bilayer. The adhesion enthalpy, in principle, provides a route to calibration of the interaction potentials against empirical data through calorimetry measurements of adhesion processes (see reference 41 ).

The ApL of the adhering bilayer in general reflects the adhesion energy, and the greatest ApL is indeed observed on the gold surface. In fact, the ApL closely matches that of a free bilayer under a tension equal to the measured value of $w$, which can be understood to mean that the gold surface is smooth at the molecular level. The separation is measured along the surface normal between the mean position of superficial atoms and the mean position of the head group atoms in the adhering leaflet. In a similar fashion, the bilayer thickness is measured along the surface normal between the mean position of the head group atoms in each leaflet. It should be noted that this definition does not allow direct comparison ${ }^{30}$ with empirical values such as those obtained from small-angle neutron scattering, but has a straightforward application here in that it allows a comparison to be made between the different surfaces in this study.

Silica surfaces subjected to adhesion simulations were modelled with silanols with the exception of the $\mathrm{Q}^{4}$ surface which had only siloxane bridges. Varying fractions $(0 \%, 9 \%$ and $18 \%$ ) of these silanol groups were ionised. In practice this means that the hydrogen site is removed from silanol group and replaced by a solvated $\mathrm{Na}$ counterion. The point of zero charge for silica (pzc) is roughly $\mathrm{pH} 2$, and the fraction of ionised silanols increases as the $\mathrm{pH}$ increases, generally to about $12 \%$ at $\mathrm{pH} 7^{35}$.

The greatest adhesion strength, $-7.5 \mathrm{mN} . \mathrm{m}^{-1}$ was registered on the amorphous surface at $0 \%$ ionisation, where the entropic contribution appeared to oppose adhesion. The effect of ionisation was to reduce the adhesion strength to $-5.3 \mathrm{mN} . \mathrm{m}^{-1}$ at $9 \%$ ionisation and further to $-4.3 \mathrm{mN} \cdot \mathrm{m}^{-1}$ at $18 \%$ ionisation where the entropic contribution now drove adhesion. The quartz surface had a relatively low adhesion strength of $-2.1 \mathrm{mN} \cdot \mathrm{m}^{-1}$ at $0 \%$ ionisation which did not change significantly at $9 \%$ ionisation, both cases entropically driven. However, at $18 \%$ ionisation no adhesion occurred at all. In contrast, the adhesion on the cristobalite surface 
increased from -3.2 to $-4.4 \mathrm{mN} \cdot \mathrm{m}^{-1}$ upon $9 \%$ ionisation, then decreased at $18 \%$ ionisation to -2.4. Each degree of ionisation on this surface appeared entropically driven, especially at $9 \%$ ionisation. With silanols replaced by siloxane bridges the same surface, now hydrophilic, registered an entropically opposed adhesion strength of only $-4 \mathrm{mN} \cdot \mathrm{m}^{-1}$.

\begin{tabular}{llllllllll} 
Formula & Polymorph & $\begin{array}{l}\text { M. } \\
\text { index }\end{array}$ & $\%$ ion & $k_{w} \mathrm{mN} \cdot \mathrm{m}^{-1}$ & $\begin{array}{l}\Delta H_{a d h} \\
\mathrm{mN} \cdot \mathrm{m}^{-1}\end{array}$ & $\begin{array}{l}\mathrm{T} \Delta S \\
\mathrm{mN} \cdot \mathrm{m}^{-1}\end{array}$ & $\begin{array}{l}\mathrm{ApL} \\
\mathrm{nm}\end{array}$ & $\begin{array}{l}\text { sep } \\
\mathrm{nm}\end{array}$ & $\begin{array}{l}\text { thick } \\
\mathrm{nm}\end{array}$ \\
\hline $\mathrm{Au}$ & $\mathrm{Fcc}$ & 111 & - & $-42(2)$ & $-118(7)$ & $-76(9)$ & 0.84 & 0.36 & 2.83 \\
$\mathrm{Au}$ & $\mathrm{F}$ & 111 & - & $-40.7(4)$ & $-77.8(6)$ & $-37(1)$ & 0.85 & 0.47 & 2.83 \\
$\mathrm{Au}^{\mathrm{b}}$ & $\mathrm{Fcc}$ & 111 & - & $-21.6(6)$ & $-93(10)$ & $-71(11)$ & 0.66 & 0.77 & 3.49 \\
$\mathrm{SiO}_{2}$ & quartz & 100 & 0 & $-2.1(4)$ & $2.1(12)$ & $4.2(16)$ & 0.65 & 0.76 & 3.15 \\
$\mathrm{SiO}_{2}$ & cristobalite & $10 \overline{1}$ & 0 & $-3.2(4)$ & $-2.8(17)$ & $0.4(21)$ & 0.67 & 0.54 & 3.08 \\
$\mathrm{SiO}_{2}$ & amorphous & - & 0 & $-7.5(5)$ & $-10.0(11)$ & $-2.5(16)$ & 0.71 & 0.52 & 2.98 \\
$\mathrm{SiO}_{2} \mathrm{c}$ & cristobalite & $10 \overline{1}$ & - & $-2.0(3)$ & $-3.4(21)$ & $-1.4(24)$ & 0.64 & 1.01 & 3.18 \\
$\mathrm{SiO}_{2}$ & quartz & 100 & 9 & $-2.5(4)$ & $3.0(16)$ & $5.5(20)$ & 0.65 & 0.76 & 3.17 \\
$\mathrm{SiO}_{2}$ & cristobalite & $10 \overline{1}$ & 9 & $-4.4(7)$ & $10.7(13)$ & $15.1(19)$ & 0.65 & 0.69 & 3.18 \\
$\mathrm{SiO}_{2}$ & amorphous & - & 9 & $-5.3(4)$ & $-4.4(11)$ & $0.8(15)$ & 0.66 & 0.93 & 3.14 \\
$\mathrm{SiO}_{2}$ & cristobalite & $10 \overline{1}$ & 18 & $-2.4(1)$ & $2.8(20)$ & $5.2(2.1)$ & 0.62 & 0.74 & 3.23 \\
$\mathrm{SiO}_{2}$ & amorphous & - & 18 & $-4.3(2)$ & $4.4(14)$ & $8.7(16)$ & 0.63 & 0.88 & 3.21 \\
$\mathrm{TiO}_{2}$ & Rutile & 110 & - & $-1.8(2)$ & $1.6(10)$ & $3.5(12)$ & 0.62 & 1.18 & 3.20 \\
$\mathrm{TiO}_{2}$ & Rutile & 100 & - & $-3.6(5)$ & $2.6(11)$ & $6.1(16)$ & 0.65 & 0.89 & 3.03 \\
$\mathrm{TiO}_{2}$ & Rutile & 101 & - & $-4.0(5)$ & $1.4(14)$ & $5.4(20)$ & 0.66 & 0.85 & 3.04 \\
$\mathrm{TiO}_{2}$ & Rutile & 001 & 0 & $-0.3(1)$ & $1.9(13)$ & $2.2(15)$ & 0.62 & 0.96 & 3.16
\end{tabular}




$\begin{array}{llllllllll}\mathrm{TiO}_{2} & \text { Anatase } & 101 & - & -1.0(4) & -6.5(14) & -5.5(18) & 0.64 & 1.00 & 3.08 \\ \mathrm{TiO}_{2} & \text { Anatase } & 100 & - & -1.1(7) & -3.7(12) & -2.6(18) & 0.63 & 1.53 & 3.22 \\ \mathrm{TiO}_{2} & \text { Anatase } & 001 & 0 & -0.6(2) & -1.7(15) & -1.2(16) & 0.64 & 1.10 & 3.14 \\ \mathrm{TiO}_{2} & \text { Anatase } & 110 & - & -4.0(3) & -13.8(23) & -9.8(25) & 0.64 & 0.81 & 3.12\end{array}$

Table 1. Results from atomistic adhesion simulations using the ribbon geometry. Numbers in brackets denote the uncertainty expressed in terms of the least significant digit. Where no error is recorded the uncertainty is smaller than the least significant digit. ${ }^{\text {a }}$ bilayer mixture with no cholesterol. ${ }^{b}$ bilayer mixture with cholesterol. ${ }^{c} Q^{4}$ surface silanols have been condensed to form siloxane bridges. With the exception of $A u^{a, b}$ data taken from our published work.

Although bilayers spontaneously adhered to all the titania surfaces there was a marked amount of variation in the magnitude of the adhesion strength. The adhesion was particularly strong on the rutile 101 and anatase 110 surfaces at $-4.0 \mathrm{mN} \cdot \mathrm{m}^{-1}$ with rutile 100 registering $3.6 \mathrm{mN} . \mathrm{m}^{-1}$. The rutile 001 and anatase 001 surfaces, on the other hand, exhibited relatively weak adhesion with values of only -0.3 and $-0.6 \mathrm{mN} \cdot \mathrm{m}^{-1}$, respectively. Curiously, the entropic contribution appeared to oppose adhesion on the anatase surfaces but to drive adhesion on the rutile surfaces.

In general, the ApL of the adhering bilayer was a reasonably accurate predictor of the adhesion strength, and estimation of this property could feasibly be employed to offer a less computationally expensive method of gauging the adhesion strength, although the manual labour required to set up the simulation is the much the same as that for the adhesion measurement used here. The thickness is a less accurate predictor and the separation even worse.

These data are for pure membranes of DMPC. However we have also simulated ${ }^{36}$ a mixed membrane containing 50\% POPC, 25\% DOPE, 15\% Bis(monoacylglycero)phosphate BMP ${ }^{37}$, and $10 \%$ sphingomyelin ,SM $(n=18)$ with and without cholesterol (in equal molar quantities to POPC when present) in contact with a gold surface. As can be seen from table 1, the adhesion strength on gold is the same as for DMPC within the error, indicating that the choice of lipid in a liposome may not be a determining factor in NP wrapping and the results in table 1 may be applicable more generally. Note however that the adhesion behaviours changes radically on the addition of cholesterol. The adhesion strength halving, so that liposomes with cholesterol will be much more difficult to penetrate, however at $\mathrm{Kw} \sim 20$ the minimum diameter for adhesion is still $0 \mathrm{~nm}$ (section 3.2) and so gold will always attach to the liposome even with cholesterol present.

\subsection{EXTENSION OF THIN FILM THEORY TO BILAYERS}

The process by which a CG bilayer disk wraps spherical particles is captured in figure 14 . Particles of diameter 6,10 and $15 \mathrm{~nm}$ were used, with a scaling factor of $8 \%$ for the particlelipid interaction corresponding to a value of $-10 \mathrm{mN} . \mathrm{m}^{-1}$ for $k_{w}$. The pull groups at the periphery of the disk were restrained at a fixed distance, $R$, from the centre while the particle was introduced. The distance along the disk normal between the peripheral pull groups and the particle centre, the penetration depth, $d$, was monitored, as was the membrane tension determined by the restraint force on the peripheral pull groups. Once the system has equilibrated and these properties have been established, $R$ is decreased and the and the system once again allowed to equilibrate. Note that as the particle is increasingly wrapped the penetration depth becomes more negative. 


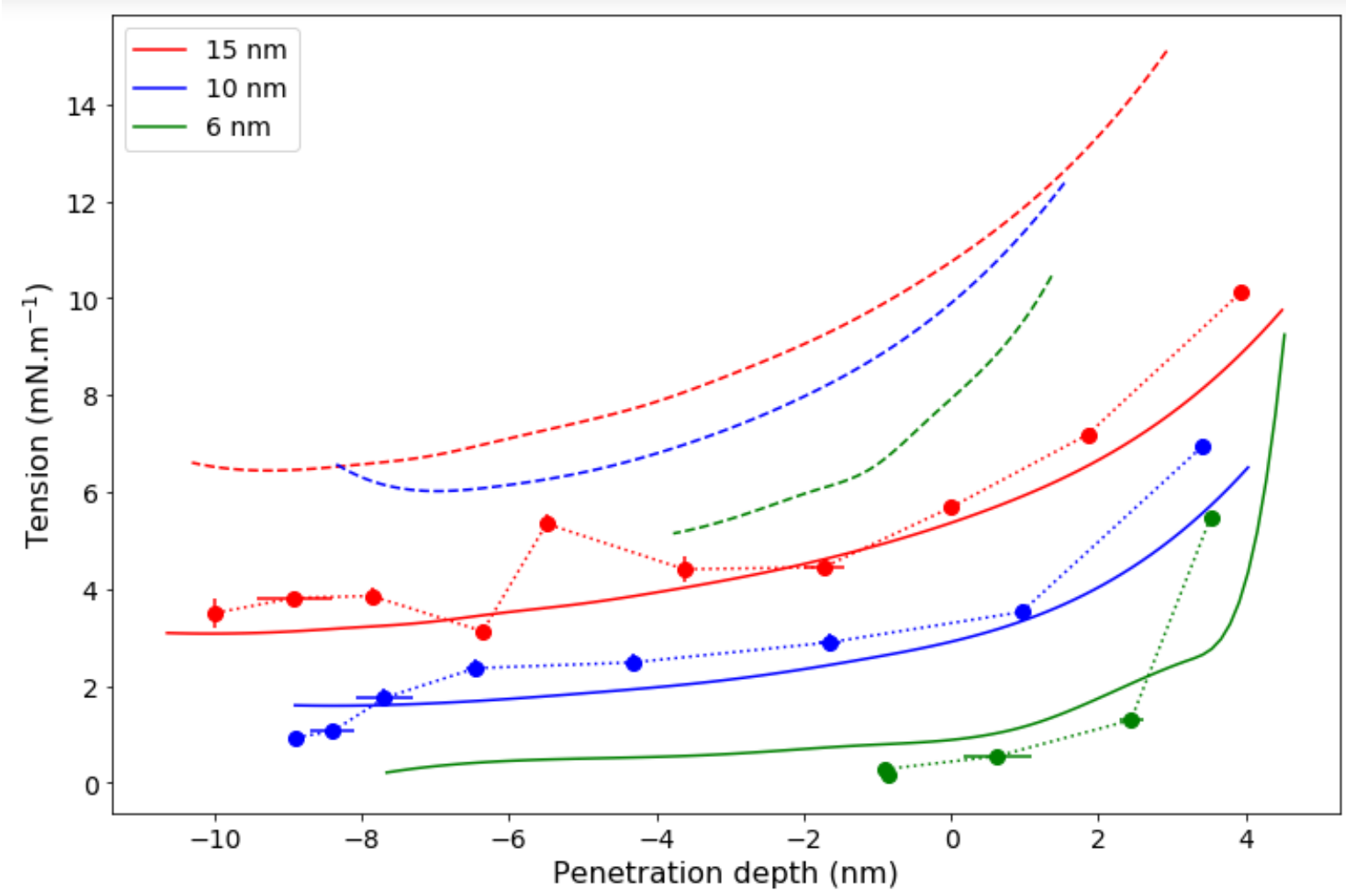

Figure 14. Membrane tension as a function of penetration depth $d$ for wrapping of spherical particles by a CG bilayer in the disk geometry. Dashed lines denote elastic theory, solid lines are fits using thin membrane theory with $w^{s}$ allowed to vary.

The dashed lines in fig 14 show the application of the modified thin film theory outlined in the methodology using the measured membrane elasticity and bending moduli and using the form of equation 6 for $w^{s}$ with the measured value of $-k_{w}$ i.e. $10 \mathrm{mN} \cdot \mathrm{m}^{-1}$. The radius was taken to be the nominal particle radius plus half the bilayer width $(2 \mathrm{~nm})$. In other words, the bilayer was modelled as a thin elastic sheet acting through the centre of the bilayer. Clearly, the theory predicts much higher tensions than were observed in simulation. Intuitively, two reasons for this discrepancy arising from the assumption of the infinitesimally thin film assumption suggest themselves. The first is that $w$ is not necessarily equal to $-k_{w}$ when the membrane thickness is finite and secondly the bending modulus of the adhering portion of the bilayer is not necessarily the same as that of a free bilayer.

Next, the theory was applied with $w^{s}$ allowed to vary as a fitting parameter. The resulting fits are shown as solid lines in 


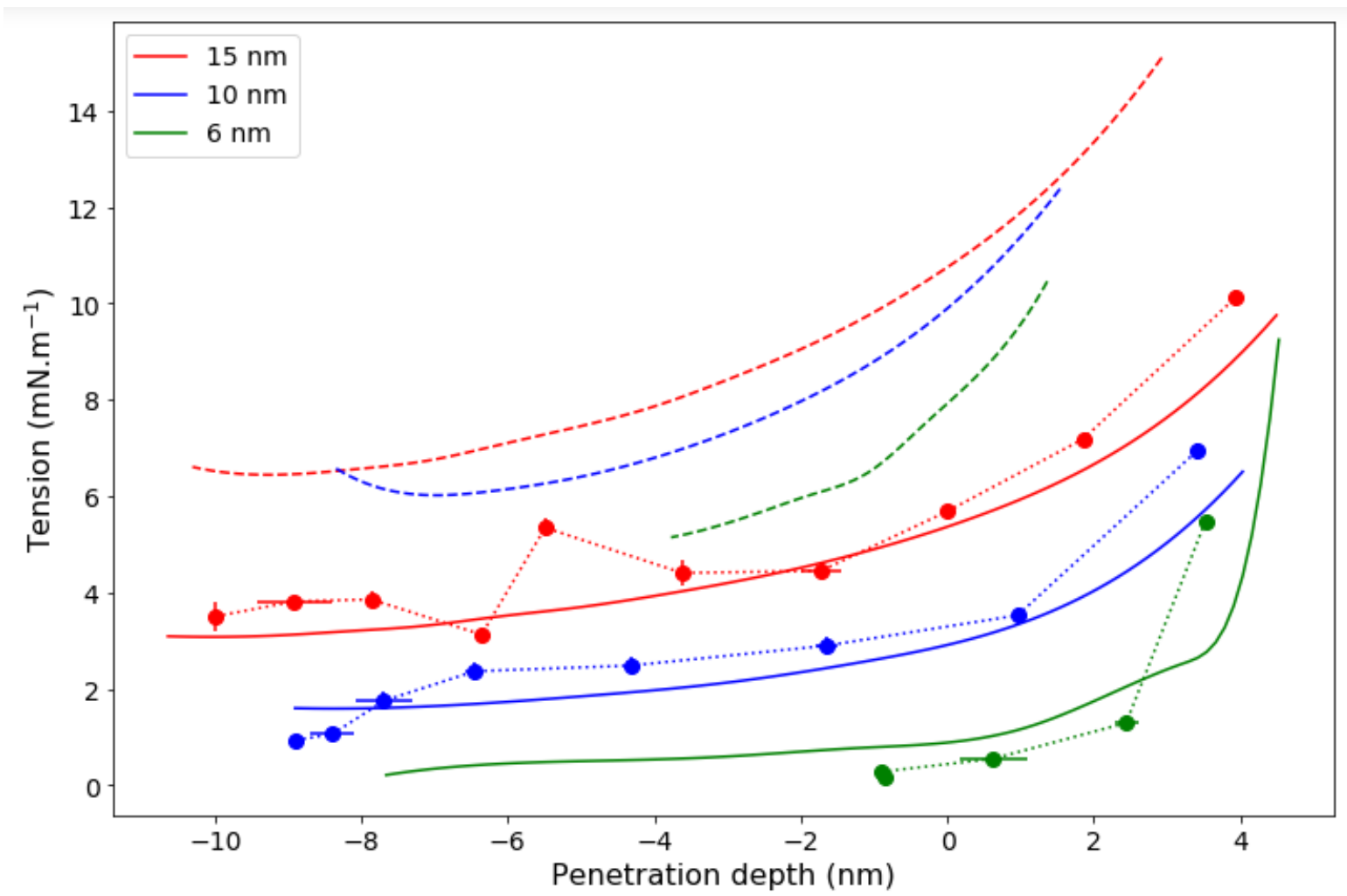

Figure 14. Note that the optimisation was performed on the dependence of membrane tension on disk radius, and the penetration depth only calculated once the full profile had been determined.

The parameter $w_{S L B}^{c}$ was also determined for cylinders of $6,10,15,20$ and $25 \mathrm{~nm}$ diameter using the simulation geometry depicted in figure 13 . These values were used to calculate $w_{S L B}^{c}$ using equation 18 In Figure 15, the values of $w_{S L B}$ are plotted against curvature in orange for cylinders and for spheres in blue. A quadratic fit is also shown in black. The intercept is fixed at $10 \mathrm{mN} \cdot \mathrm{m}^{-1}$, the measured value of $-k_{w}$ ensuring that in the limit of vanishing curvature the flat surface adhesion strength is recovered. The value for $p_{1}$ is -5.46 $\times 10^{-11} \mathrm{~N}$ and $p_{2}$ is equal to $-2.71 \times 10^{-20} \mathrm{~J}$.

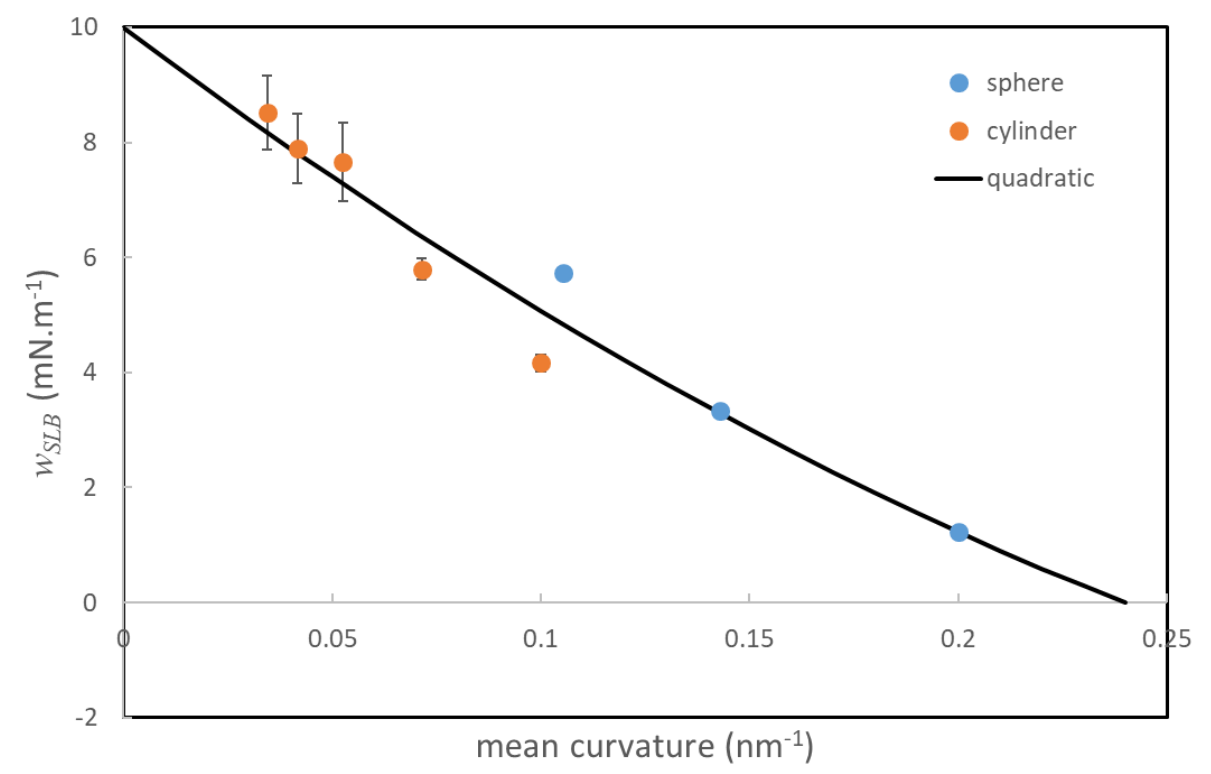

Figure 15. The effective contact energy, $w_{S L B}$, as a function of curvature. Blue points denote spheres and orange points denote cylinders. The black line is a quadratic fit to both sets of data. 
In Figure 16 the wrapping behaviour of a sphere, cylinder and cube are shown. Each shape is $10 \mathrm{~nm}$ in diameter. The behaviour predicted by thin film theory is also shown for the sphere and cylinder. Cube wrapping was performed with the same simulation geometry as the sphere.

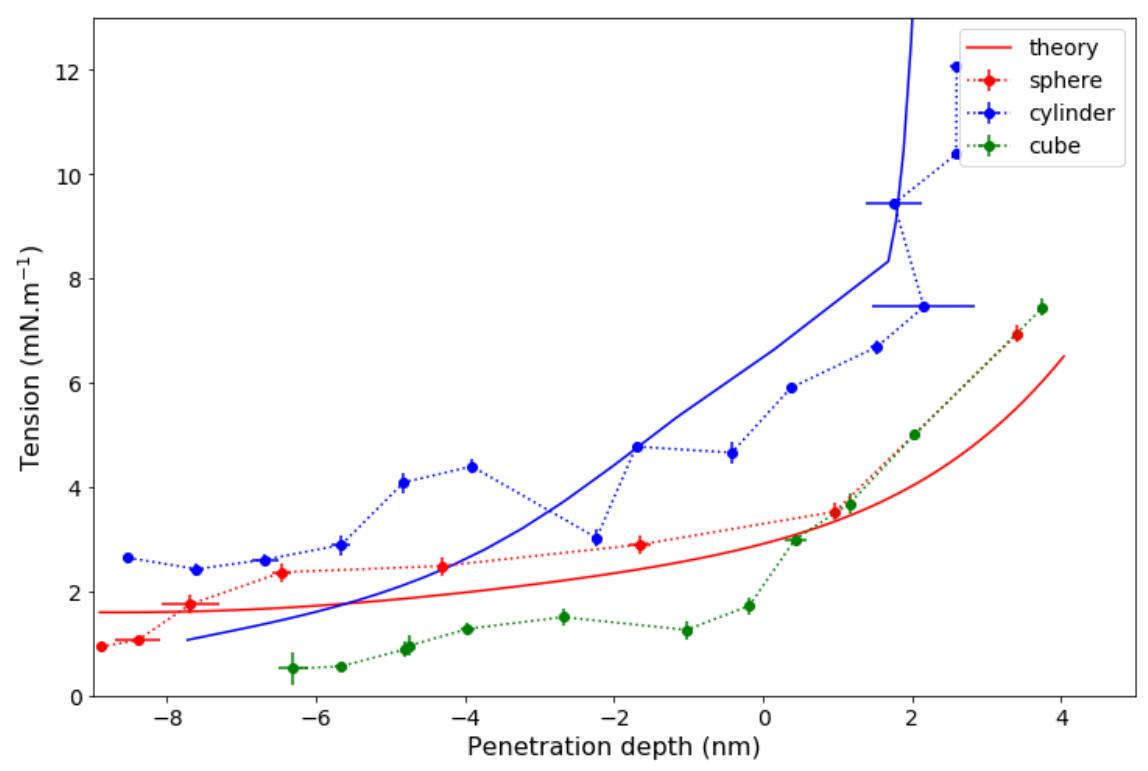

Figure 16. Tension vs penetration depth for a sphere (red), cylinder (blue) and cube (green) $10 \mathrm{~nm}$ in diameter. Solid lines show predictions from theory, using modified values of $w$.

\subsection{PREDICTION OF WRAPPING BEHAVIOUR}

A particle of a given material will adhere to a membrane as long as $w_{S L B}$ is positive (see equation 15 and 16). Equivalently, a particle will adhere if the particle size is greater than a critical diameter. In the table below we list estimates of the critical diameter for materials in this study. In the case of titania, values are listed for the spherical and cylindrical case for each polymorph which combine the adhesion strengths measured on the different cleavage planes listed in Table 1. The adhesion strength for spherical particles was calculated by taking the weighted average of adhesion strength across all four cleavage planes, where the weighting was according to the inverse of the surface energy for the plane, as calculated from DFT simulations ${ }^{38}$. A similar procedure was followed for the cylindrical case, except that the 110 plane was excluded. Note that all the other planes are perpendicular to the $b_{2}$ lattice vector and can thus in combination can form a low surface energy cylinder

\begin{tabular}{lllllll} 
Formula & Polymorph & $\begin{array}{l}\text { M. } \\
\text { index }\end{array}$ & $\%$ ion & $\begin{array}{l}k_{w} \\
\mathrm{mN} . \mathrm{m}^{-1}\end{array}$ & $\mathrm{Nm}$ & $\begin{array}{l}d_{c r i t}^{s} \\
\mathrm{~nm}\end{array}$ \\
\hline $\mathrm{Au}$ & $\mathrm{Fcc}$ & 111 & - & $-42(2)$ & 0 & 0 \\
$\mathrm{SiO}_{2}$ & quartz & 100 & 0 & $-2.1(4)$ & 46.0 & 21.0 \\
$\mathrm{SiO}_{2}$ & cristobalite & $10 \overline{1}$ & 0 & $-3.2(4)$ & 28.1 & 12.0
\end{tabular}




$\begin{array}{lllllll}\mathrm{SiO}_{2} & \text { amorphous } & - & 0 & -7.5(5) & 8.2 & 2.1 \\ \mathrm{SiO}_{2}{ }^{*} & \text { cristobalite } & 10 \overline{1} & - & -2.0(3) & 48.6 & 22.3 \\ \mathrm{SiO}_{2} & \text { quartz } & 100 & 9 & -2.5(4) & 37.6 & 16.8 \\ \mathrm{SiO}_{2} & \text { cristobalite } & 10 \overline{1} & 9 & -4.4(7) & 18.7 & 7.3 \\ \mathrm{SiO}_{2} & \text { amorphous } & - & 9 & -5.3(4) & 14.4 & 5.2 \\ \mathrm{SiO}_{2} & \text { cristobalite } & 10 \overline{1} & 18 & -2.4(1) & 39.5 & 17.8 \\ \mathrm{SiO}_{2} & \text { amorphous } & - & 18 & -4.3(2) & 19.3 & 7.6 \\ \mathrm{TiO}_{2} & \text { Rutile } & \text { Sphere } & - & -2.48 & 38.0 & - \\ \mathrm{TiO}_{2} & \text { Anatase } & \text { Sphere } & - & -1.47 & 68.3 & - \\ \mathrm{TiO}_{2} & \text { Rutile } & \text { Cylinder } & - & -2.89 & - & 13.9 \\ \mathrm{TiO}_{2} & \text { Anatase } & \text { Cylinder } & - & -1.11 & - & 44.2\end{array}$

Table 2. Predicted critical diameters for a range of materials for both cylindrical and spherical particles. * This is the dehydroxylated surface corresponding to heat treatment where all surface oxygen are bridging. $d_{c r i t}=2^{*}$ nominal nanoparticle radius $r$.

According to thin film theory, if the membrane remains tensionless a particle will be completely wrapped if it exceeds the critical diameter. The outer membranes of many cells have lipid reservoirs that serve to regulate the tension, in contrast, when a particle interacts with a liposome or a simple cell, such as a red blood cell, there are no such cellular mechanisms and the tension may rise as the membrane is deformed, particularly when the particle is large compared to the liposome or when many particles interact simultaneously ${ }^{39}$. If the tension rises significantly it may halt the wrapping process before completion. Note that wrapping is defined to be complete when the free part of the membrane makes contact with itself and that this typically occurs when the angle $\alpha$ is less than $180^{\circ}$, since the contact occurs at the inflection point on the membrane rather than the surface. With membranes of finite thickness, the angle at complete wrapping is less than the thin film model, as illustrated inError! Reference source not found. fig 17 .

Figure 17: Illustration of the finite membrane model showing a cross section schematic of a complete wrapped spherical particle. The particle, $20 \mathrm{~nm}$ in diameter, is shown in yellow, the midplane of the bilayer is red and the hydrophilic surfaces of the bilayer are blue.

Note that that the outer edges of the bilayer, shown in blue, are in contact while the midplane, in red, equivalent to the thin film model, is not.

Applying this criterion, the maximum tension at which compete wrapping can occur can be calculated. For a given tension, the total energy of the system, was calculated as a function of the wrapping angle, $\alpha$. The energy comprises the contact energy which drives the wrapping process and the bending and tension energy components of the free membrane which opposes it. In the case of spherical particles, the free membrane profile was solved numerically following the procedure detailed in reference 19. For a given tension, if there exists a local minimum in the energy profile at an value of the the wrapping angle that is less than that required to complete wrapping, as illustrated by $F$, then the tension is deemed too great to allow complete wrapping. The maximum tension for complete wrapping is the greatest tension at which the local minimum in the total energy as a function of wrapping angle is equal to or 
greater than the angle for complete wrapping. The membrane profile around cylindrical particles can be determined analytically ${ }^{40}$ and the resulting energy dependence on the the wrapping angle is monotonic, therefore the maximum tension increases monotonically with cylinder diameter.

This is displayed in as a function of cylinder diameter for a range of adhesion strengths spanning those of the materials in this study. For a cylinder of given diameter, complete wrapping will occur if the tension is below the curve for the corresponding adhesion strength. If the tension is above the curve partial wrapping will occur. If the particle diameter is less than the diameter corresponding to the left most part of the curve (the critical diameter, table 2) the cylinder will not adhere. The maximum tension increases monotonically with cylinder radius and with adhesion strength. Even at the adhesion strength of gold $\left(40 \mathrm{mN} \cdot \mathrm{m}^{-1}\right)$ and large cylindrical radius the maximum tension remains below the rupture tension $\left(\sim 45 \mathrm{mN} . \mathrm{m}^{-1}\right)$. At the critical diameter for a particular adhesion strength any tension in the membrane will halt the wrapping process.

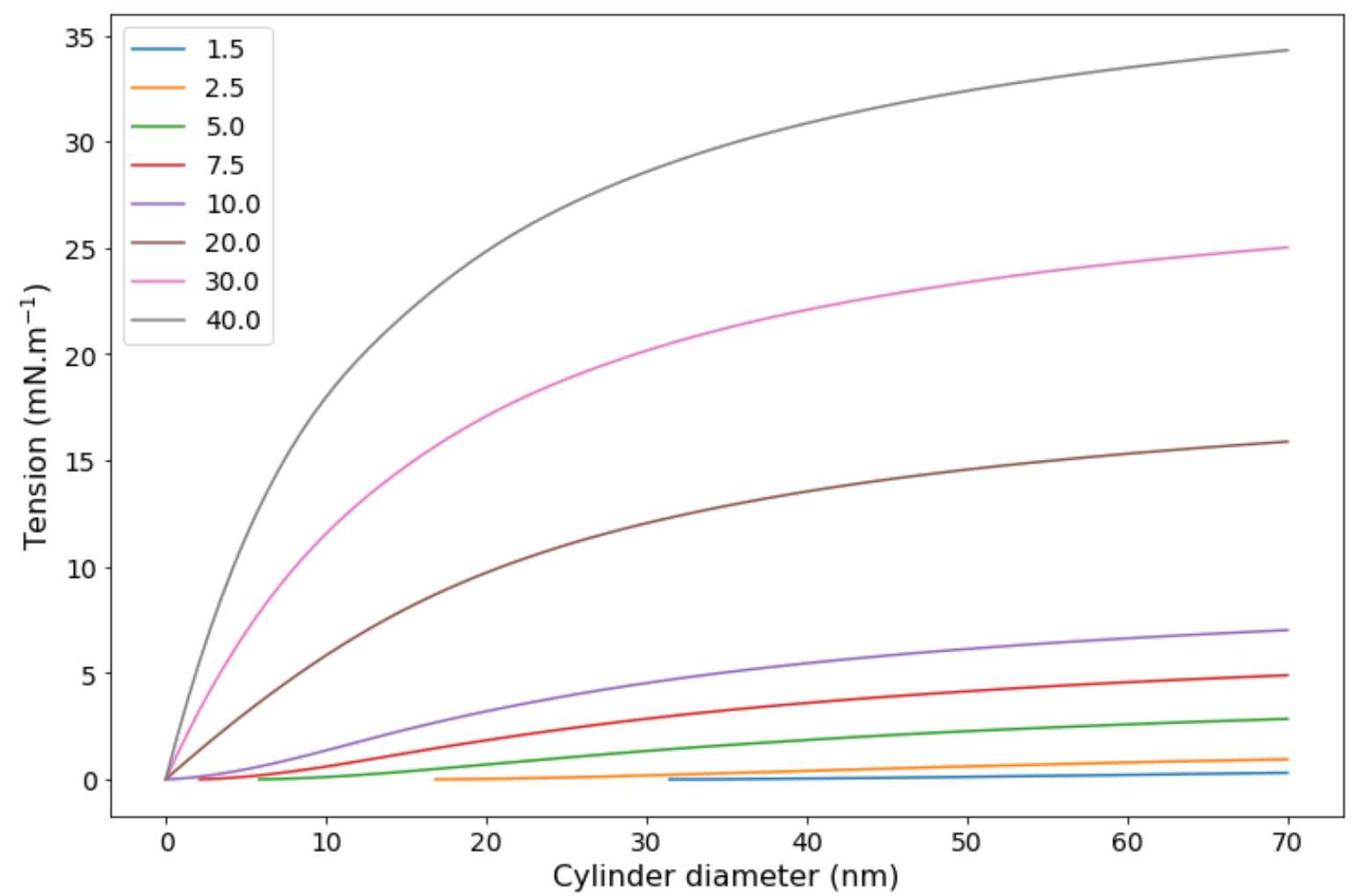

Figure 17. The maximum tension for complete wrapping of cylindrical particles as a function of diameter for a range of adhesion strengths. 
The situation for spherical particles is shown in Error! Reference source not found. 19. The maximum tension for complete wrapping of spherical particles as a function of diameter for a range of adhesion strengths.

The maximum tensions for spheres (figure 19) are much lower than for cylinders of the same radius due to the greater particle curvature and consequent induced membrane curvature. There is also a local minimum in the free membrane energy dependence on wrapping angle that prevents complete wrapping from occurring when the tension is too large. The energy barrier is largely due to tension, rather than bending energy, and the maximum tension is determined by the tension at which this energy barrier disappears. As the tension is lowered past the maximum tension, it results in a discontinuous transition from partially wrapped to completely wrapped states. For example, If the tension is say 0.5 and the diameter is 30 , then the sphere will completely wrap if the adhesion strength is $7.5 \mathrm{mN} / \mathrm{m}$ or greater. If the adhesion strength is 5 it will partially wrap and 2.5 or 1.5 will not adhere. Note that the maximum tension for a given $\mathrm{Kw}$ will always be greater than that for a smaller $\mathrm{Kw}$ at the same diameter.

If we consider a system of liposomes in a solution of particles it is possible to use these data to determine a maximum particle surface concentration on the liposome for complete wrapping to occur. At greater particle concentrations only partial wrapping will occur as the membrane deformation induced by the particles will increase the tension beyond the maximum before complete wrapping can occur. Assume that initially the liposomes are tensionless with an area per lipid $A p L_{0}$ which can be read from the atomistic simulation data shown in Figure 9 and that each particle consumes an area of bilayer equal to $4 \pi a^{2}$ at a tension equal to the maximum tension indicated in Error! Reference source not found.. The ApL at the maximum tension $A p L_{\max }$ can also be estimated from the atomistic data and we can write the following equation for the maximum particle concentration $c_{\text {max }}$ expressed in particles per square $\mathrm{nm}$ of the liposome surface:

$$
c_{\max }^{\text {sphere }}=\frac{\left({ }^{\left.A p L_{\max } / A p L_{0}-1\right)}\right.}{4 \pi a^{2}}
$$

The maximum particle concentration for complete wrapping of all nanoparticles at the liposome surface is plotted on a log scale in fig 19. 




Figure 18. Maximum spherical particle concentration, expressed in particles per square $\mathrm{nm}$ of bilayer, for complete wrapping over a range of values of adhesion strengths.

In the cylindrical case we can write a similar equation expressing the maximum particle concentration in terms of the length of cylinder per unit area of liposome surface:

$$
c_{\max }^{c y l}=\frac{\left(A p L_{\max } / A p L_{0}-1\right)}{2 \pi a}
$$

Application of this equation results in fig 20 In this case the length of cylinder per unit area that can be completely wrapped by a liposome increases with cylinder diameter at low adhesion strength and decreases at high adhesion strength. 


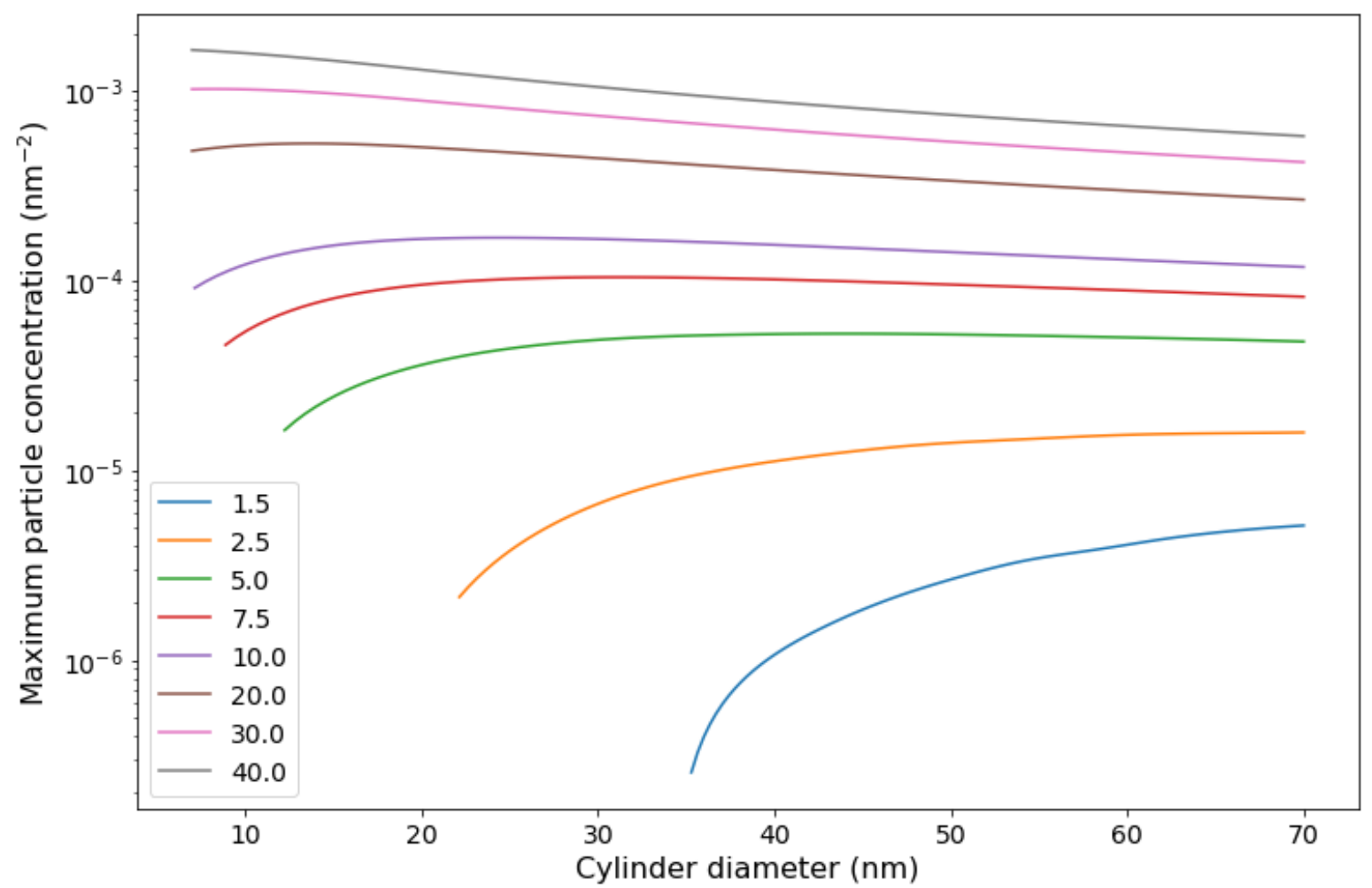

Figure 19. Maximum cylindrical particle concentration, expressed in length of cylinder per square $\mathrm{nm}$ of bilayer, for complete wrapping over a range of values of adhesion strengths.bmp

\section{Discussion}

In this section the adhesion strength simulations and extension of elastic theory are discussed along with example applications of Nanolip, the code embodying the data presented in the last section.

\section{ADHESION STRENGTH}

Adhesion strength is difficult to measure experimentally, which is why the ribbon geometry simulation, discussed above, is valuable. However, the estimate of adhesion strength is only as accurate as the classical force fields used. The Slipids forcefield for DMPC has been tested extensively and is shown to be a reliable predictor of many properties of free bilayers. That is, the lipid-lipid and lipid-water interactions have been finely tuned based on empirical data and DFT simulations. Likewise, the substrate models have been calibrated using empirical data for their interaction with water, such as adsorption enthalpy and water surface density profiles. However, the substrate-lipid interaction potentials are generated using mixing rules as there is little empirical data to calibrate the potentials with.

In the case of gold, a reliable experimental measurement of adhesion strength does exist ${ }^{34}$. The mixing rule generated interaction potential overestimated the adhesion strength by $25 \%$. Calibration of the interaction potential against the empirical adhesion strength required scaling of the well-depth by 0.925 . So, the data for the other surfaces which have been not been calibrated should probably be viewed as being accurate to within $25 \%$. In theory, calibration data could be generated by DFT simulations, but there are severe limitations on the size and timescale accessible to such simulations. A single lipid interacting with the surface could be simulated using DFT although our simulations show that the single lipid adsorption strength is not a reliable predictor of adhesion strength. On the other hand, it may help to calibrate the individual atomic interactions. Attempts to generate calibration data with calorimetry measurements of particles interacting with vesicles have so far been beset with difficulties, 
and it is apparent that the adsorption enthalpies of the metal oxides in this study are too small to be measured reliably with current instruments ${ }^{41}$. However microscopy of gold nanoparticles in contact with liposomes display behaviours consistent with the analysis presented here ${ }^{41}$.

From figure 1 there is considerable variation in adhesion strength for different cleavage planes of the materials considered in this study. In order to accurately model nanoparticles it would be best if accurate experimental evidence of the structure of the particle surface were known. In the absence of such evidence it was assumed, in the case of titania, that the particle surface consists of a combination of cleavage planes with the lowest surface energies as determined by DFT simulation. Intuitively, this assumption appears justified when the particle is formed in conditions close to the physiological environment in a process near to chemical equilibrium, say by crystallisation from solution. On the other hand, where the particle is formed in very different condition far from equilibrium it may be that surfaces with altogether different structures may be favoured. Indeed, other groups ${ }^{42}$ have produced convincing evidence that anatase Titania cylinders with diameters far below the critical diameter calculated here adhere to liposomes and even become fully wrapped.

The translation of the adhesion strengths measured for the individual cleavage planes into a single value that is used to try to capture the behaviour of cylindrical and spherical particles could also be improved by using a modified form of the Wolff construction weighted to provide the desired particle morphology at a minimum surface energy. The assumption that the average of the surface plane adhesion energy reflects the wrapping behaviour can also be tested in CG simulations by using particles with different adhesion strengths on the relevant cleavage planes.

The essence of the modification made to thin film theory is replacing the contact energy with an effective contact energy which operates on a surface with a radius $2 \mathrm{~nm}$ greater than the nominal particle radius. The effective contact energy is related to the adhesion strength by an empirically derived second order polynomial function of curvature. There is no real theoretical basis for the modification, but one could possibly be developed based on microscopic considerations of the particle-bilayer interface. It should be noted that only one value of the adhesion strength has been utilised in simulations so far and it may well be that the parameters vary significantly with adhesion strength.

The other main assumption is that the free bilayer behaves like a thin sheet acting through the midplane of the bilayer, in so far as the bending energy is concerned. For the larger particles at moderate degrees of wrapping this assumption appears justified. However, the free bilayer curvature around the $6 \mathrm{~nm}$ particle is rather high. Intuitively we might expect this assumption to break down here and indeed of all three particles sizes modelled the theoretical prediction of tension as a function of penetration is least accurate for the $6 \mathrm{~nm}$ particle. This suggests a possible improvement to the model by imposing an extra energy penalty for large curvature of the free bilayer. Complete wrapping has yet to be achieved in simulation and from thin film theory large curvatures are expected here also.

The effectiveness of the overall approach can be tested against the predictions of maximum $\begin{array}{lllll}\text { particle concentration } & \text { in } & \text { fig } & 19 & \text { and }\end{array}$ 


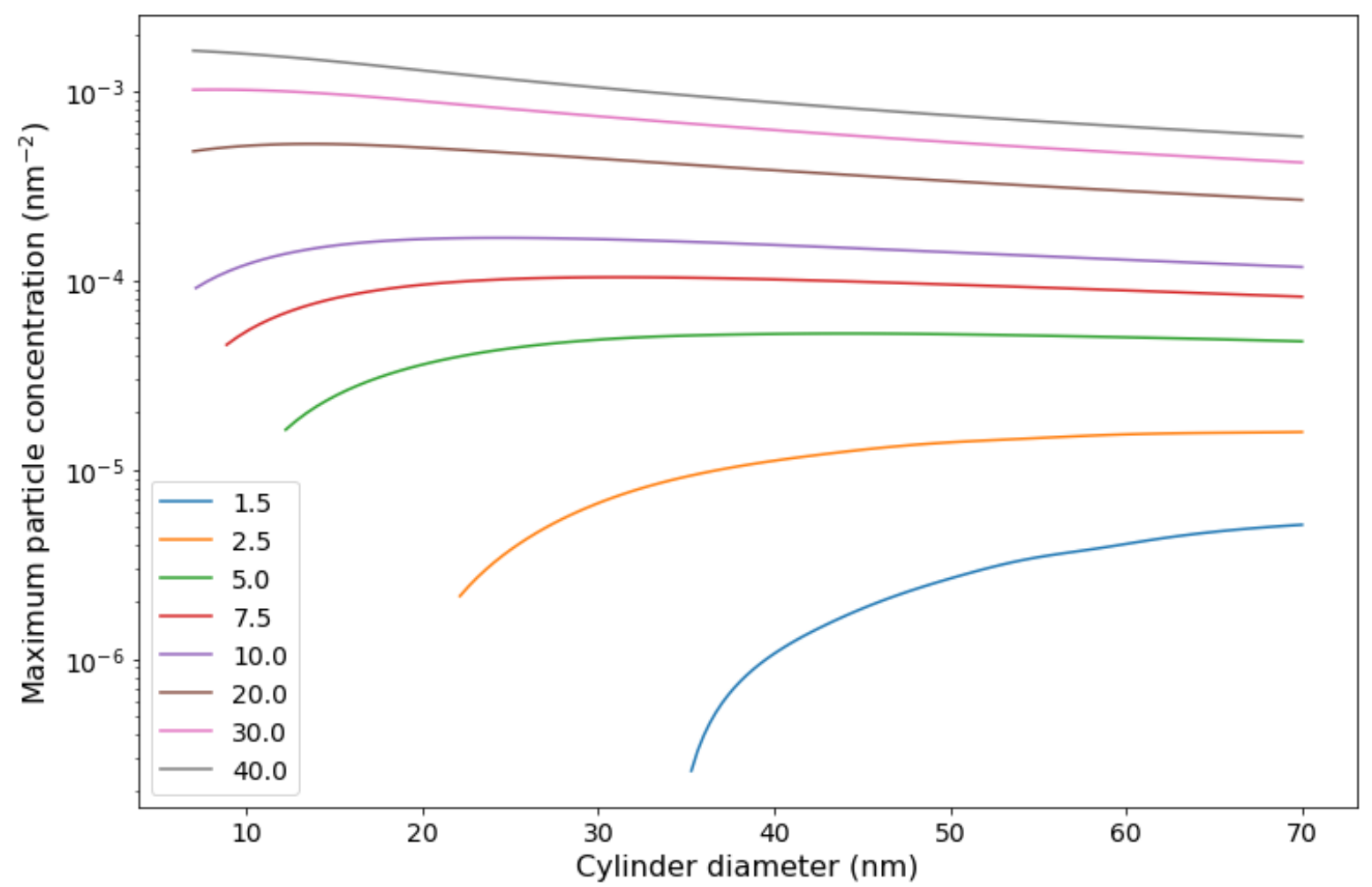

Figure 1920 by exposing particles of known adhesion strength at varying concentration to liposomes and observing the extent of wrapping using an appropriate form of microscopy.

We now consider two applications of our model; predicting critical diameters, maximum membrane tension and nanoparticle concentrations at the maximum membrane tension.

Toxicology

As discussed in the introduction most toxicity pathways involve an uptake of nanoparticles by the cell, either via active endocytosis or by a passive process. The probability of uptake is determined by the nanoparticle physicochemical properties and by the interaction between the nanoparticle and the cell membrane. In the preceding sections we described a mechanistic model of nanoparticle uptake by model cell membranes, the liposomes, and used simulations to predict the appropriate physical property data for a range of materials. Based on these calculations we can calculate critical sizes and concentrations for nanoparticle uptake allowing nanoparticles to be classified as either those that a) penetrate the cell b) adhere to the cell or c) do not adhere to the cell, if the nanoparticle exists in its native state at the point of contact (for the moment we do not have estimates of $\mathrm{Kw}$ for lipid membrane/protein corona interactions, while for citrate stabilised gold nanoparticles we assume the citrate is displaced by the membrane upon adhesion). Unlike GUVs, many cells maintain a plasma membrane reservoir ${ }^{43,44}$ to buffer against changes in membrane tension so that changes in the membrane tension will not increase as described by equation 19 . In this case only the critical diameter can be used to predict nanoparticle uptake, as penetration of the cell membrane will not lead to tension mediated inhibition of wrapping. The fact that cells have diameters in the 10's of micron range (blood cells, lymphocytes) and can also have locally planar surfaces (endothelial, muscle...) means that the approximations made in section 3 in describing the free energy effects of membrane curvature are likely to hold.

Cases where the nanoparticles are covered in protein (this is the case in vivo but not always in vitro where table 2 then applies) are not covered in table 2: our work needs to be extended 
through simulations of lipid membranes on corona proteins such as serum albumin. However once through the plasma membrane, nanoparticles with corona will be taken up by lysosomes inside the cell and the protein removed in the acid environment of the lumen exposing the nanoparticle surface. In this regard the values of $\mathrm{Kw}$ in table 2 allow us to rank the nanoparticles with respect to the strength of the interaction with the lipids forming the inside of the lysosome (or more accurately the autolysosome) membrane. We can see that gold nanoparticles should interact strongly with these lipids, with potential for membrane disruption while silica and titania have a much weaker interaction indicating they may well not be able to disrupt the membrane, for example by lysosomal membrane permabilisation or rupture and release of lysosomal hydrolases from the lumen into the cytosol and subsequent toxic effects. Further work is required to extend our models to include the effect of membrane protein in the autolysosome, and the effect of negative membrane curvature as the cell vesicles have diameters $\sim 500 \mathrm{~nm}$ comparable to the LUVs discussed in the next section.

\section{Separations}

The code can be used to design separations process based on nanoparticles adhering to liposomes and the liposomes subsequently separated from solution, for instance by centrifugation. We assume the process is wrapping limited so that diffusion of nanoparticles to the liposome is much faster than the nanoparticle wrapping process. For gold particles in contact with liposomes with and without cholesterol, Nanolip gives the minimum diameter for adhesion to a liposome as zero, which we take as a indication that most gold nanoparticles will adhere. Hence any system containing gold particles mixed with non gold species having diameters $<\mathrm{d}_{\text {crit }}(\mathrm{i})$ as given in table 2 will immediately separate into two components, gold loaded liposomes and a solution of non-adhering species. If the liposomes can be separated from solution (i.e. by centrifugation) then they will only contain gold.

In the absence of a species for which dcrit 0 (here gold) then a clear divide exists between those species in solution with $d<d c r i t$ and those with $d>d c r i t$, the former not adhering to the liposomes while the latter will adhere.

Note that we have extended thin film theory to finite-thickness membranes simply by modifying the adhesion strength by adding a curvature dependent term. We have inferred the effective adhesion strength of curved surfaces from the tension of the free part of the bilayer during the initial stages of wrapping when the free bilayer is not highly curved. The modified adhesion strengths are used with thin film theory to predict a maximum tension which will halt the wrapping process before complete wrapping can occur. A simple particle-vesicle model, as described by Dietrich et. al. ${ }^{45}$, was used to estimate how many particles can be engulfed before reaching this tension. This stage of the treatment could be made more rigorous by employing the approach of Deserno and Gelbert ${ }^{46}$ which incorporates a more accurate model of the nanoparticle-vesicle complex.

In effect, the modification of thin film theory described here accounts for the difference in bending behavior between infinitesimally thin films and films of finite thickness, but is only applied to the SLB at the particle surface. The approach could be further enhanced by incorporating a curvature dependent bending energy penalty to the free part of the bilayer. This would serve to further limit the predicted uptake of smaller particles, which generate large degrees of curvature in the free bilayer near complete wrapping. Also implicit in our approach is the assumption that the free membrane is flat at large distances from the particle, an assumption that breaks down when the particles size is large in relation to the size of the liposome. We can also separate by shape, cylinders (in table 2) always have a $d_{\text {crit }}$ (cylinder) 
$<0.5 \mathrm{~d}_{\text {crit }}$ (sphere). A solution of spheres and cylinders would separate into cylinders inside the GUV and spheres in solution.

\section{CONCLUSIONS}

Adhesion strength estimates based on atomistic classical molecular dynamics simulation for a variety of materials representing a range of strengths are presented. An extension to thin film theory was advanced that captures the wrapping behaviour of both cylindrical and spherical particles by bilayers of finite thickness, observed in coarse grain simulations, using a single pair of parameters. The flat surface adhesion strength is recovered in the limit of large particle radius. The modified theory was applied to generate diagrams that allow the prediction of the extent of wrapping based on adhesion strength, membrane tension and particle concentration and hence the classification of NPs according to their ability to penetrate membranes. The results of section 3 have been interpolated and will be available in the code 'Nanolip' available from http://www.smartnanotox.eu/?page id=143 in due course.

\section{ACKNOWLEDGMENTS}

We acknowledge funding from the European Union's Horizon 2020 research and innovation programme under Grant Agreement No. 686098, "Smartnanotox" and access to supercomputing facilities though the ARCHER UK National Supercomputing Service.

\section{REFERENCES}

\footnotetext{
${ }^{1}$ Sajid M, llyas M, Basheer $\mathrm{C}$, et al. Impact of nanoparticles on human and environment: review of toxicity factors, exposures, control strategies, and future prospects. Environ Sci Pollut Res. 2015;22(6):4122-4143.

2 Joris F, Manshian BB, Peynshaert K, et al. Assessing nanoparticle toxicity in cell-based assays: influence of cell culture parameters and optimized models for bridging the in vitroin vivo gap. Chem Soc Rev. 2013;42(21):8339-8359.

${ }^{3}$ Lewinski N, Colvin V, Drezek R. Cytotoxicity of nanoparticles. Small. 2008;4(1):26-49.

${ }^{4}$ Yildirimer L, Thanh NTK, Loizidou M, et al. Toxicology and clinical potential of nanoparticles. Nano Today. 2011;6(6):585-607.

${ }^{5}$ Yamashita K, Yoshioka Y, Higashisaka K, et al. Silica and titanium dioxide nanoparticles cause pregnancy complications in mice. Nat Nanotechnol. 2011;6(5):321-328.

${ }^{6}$ Gottschalk F. Nowack B. The release of engineered nanomaterials to the environment.J Environ Monit. 2011;13(5):1145-1155.

7 Nel A, Xia T, M€adler L, et al. Toxic potential of materials at the nanolevel. Science. 2006;311(5761):622-627.

${ }^{8}$ Ian Rouse, David Power, Erik G. Brandt, Matthew Schneemilch, Konstantinos Kotsis, Nick Quirke, Alexander P. Lyubartsev, Vladimir Lobaskin' Advanced in silico characterisation of nanomaterials for nanoparticle toxicology', http://arxiv.org/abs/2007.04017

${ }^{9} \mathrm{http}: / /$ smartnanotox.scientia.global/

10 EU NanoSafety cluster - about the NanoSafety cluster. Available from: https://www.nanosafetycluster.eu/.

${ }^{11}$ K. Kettler, K. Veltman, D. van de Meent, A. van Wezel, and A. J. Hendriks, "Cellular uptake of nanoparticles as determined by particle properties, experimental conditions, and cell type," Environ. Toxicol. Chem. 33,481-492 (2014).

${ }^{12}$ A liposome is an artificial lipid vesicle

${ }^{13}$ R. Michel, E. Kesselman, T. Plostica, D. Danino, and M. Gradzielski,"Internalization of silica nanoparticles into fluid Liposomes: Formation of interesting hybrid colloids," Angew. Chem., Int. Ed. 53, 12441-12445(2014).
} 
${ }^{14}$ Y. Roiter, M. Ornatska, A. R. Rammohan, J. Balakrishnan, D. R. Heine, and S. Minko, "Interaction of nanoparticles with lipid membrane," Nano Lett.8, 941-944 (2008).

${ }^{15}$ Knut Erik Tollefsen , 'Applying Adverse Outcome Pathways (AOPs) to support Integrated Approaches to Testing and Assessment (IATA)', 70, 2014, 629

${ }^{16}$ M Schelvan et al '..Iysosomal membrane permeabilization..', J Tox Env Health B'. 20 ,230, (2017)

${ }^{17}$ C. Pavan,B. Fubini, "Unveiling the variability of "quartz hazard" Chem. Res. Tox. 30(1), 469 , (2017).

${ }^{18}$ W. Helfrich, "Elastic properties of lipid bilayers: Theory and possible experiments," Z. Naturforsch. C 28(11), 693 (1973).

${ }^{19} \mathrm{M}$. Deserno, "Elastic deformation of a fluid membrane upon colloid binding," Phys. Rev. E 69, 031903 (2004).

${ }^{20} \mathrm{H}$ Mayaron et al,' From the roundabout of molecular events to nanomaterial-induced chronic inflammation prediction', doi: https://doi.org/10.1101/2020.02.27.966036

${ }^{21}$ P. Nativo, I A. Prior and M Brust, 'Uptake and Intracellular Fate of Surface Modified Gold Nanoparticles', ACS Nano, 2, 1639, 2008

22 C. Contini, M. Schneemilch, S. Gaisford, and N. Quirke, "Nanoparticle-membrane interactions," J. Exp. Nanosci. 13(1), 62-81 (2018).

${ }^{23}$ Schneemilch, M., \& Quirke, N. (2016). Free energy of adsorption of supported lipid bilayers from molecular dynamics simulation. Chemical Physics Letters, 664, 199-204. https://doi.org/10.1016/j.cplett.2016.10.010

${ }^{24}$ Schneemilch, M., \& Quirke, N. (2018). Free energy of adhesion of lipid bilayers on silica surfaces. The Journal of Chemical Physics, 148(19), 194704. Erratum, J. Chem. Phys., 229901 150, (2019).

${ }^{25}$ M. Schneemilch, N Quirke, Free energy of adhesion of lipid bilayers on titania surfaces ,J. Chem. Phys. 2019, 151, 134707.

26 J. P. M. Jämbeck and A. P. Lyubartsev, 'Derivation and Systematic Validation of a Refined All-Atom Force Field for Phosphatidylcholine Lipids', The Journal of Physical Chemistry B, vol. 116, no. 10, pp. 3164-3179, Mar. 2012.

27 J. P. M. Jämbeck and A. P. Lyubartsev, 'An Extension and Further Validation of an AllAtomistic Force Field for Biological Membranes', Journal of Chemical Theory and Computation, vol. 8, no. 8, pp. 2938-2948, Aug. 2012.

28 J. P. M. Jämbeck and A. P. Lyubartsev, 'Another Piece of the Membrane Puzzle: Extending Slipids Further', Journal of Chemical Theory and Computation, vol. 9, no. 1, pp. 774-784, Jan. 2013.

${ }^{29}$ Schneemilch, M., \& Quirke, N. (2016). Free energy of adsorption of supported lipid bilayers from molecular dynamics simulation. Chemical Physics Letters, 664, 199-204. https://doi.org/10.1016/j.cplett.2016.10.010

30 Schneemilch, M., \& Quirke, N. (2018). Free energy of adhesion of lipid bilayers on silica surfaces. The Journal of Chemical Physics, 148(19), 194704. Erratum, J. Chem. Phys., 229901 150, (2019).

${ }^{31}$ M. Schneemilch, N Quirke, Free energy of adhesion of lipid bilayers on titania surfaces ,J. Chem. Phys. 2019, 151, 134707.

${ }^{32}$ A Mirzoev, A P Lyubartsev, 'MagiC: Software' J of Chem Theory Computation, 9, 1512 (2013)

$33 \mathrm{M}$. Hu, P. Diggins, and M. Deserno, 'Determining the bending modulus of a lipid membrane by simulating buckling', The Journal of Chemical Physics, vol. 138, no. 21, p. 214110, 2013.

34 X. Bin, I. Zawisza, J. D. Goddard, and J. Lipkowski, 'Electrochemical and PM-IRRAS Studies of the Effect of the Static Electric Field on the Structure of the DMPC Bilayer Supported at a Au(111) Electrode Surface', Langmuir, vol. 21, no. 1, pp. 330-347, Jan. 2005.

${ }^{35}$ F. S. Emami, V. Puddu, R. J. Berry, V. Varshney, S. V. Patwardhan, C. C.Perry, and H. Heinz, "Force field and a surface model database for silica tosimulate interfacial properties in atomic resolution," Chem. Mater. 26(8), 2647-2658 (2014).

${ }^{36}$ M. Schneemilch, N Quirke, unpublished data 
${ }^{37} \mathrm{H}$ D Gallala, K Sandhoff, Biological function of the cellular lipid BMP-BMP as a key activator for cholesterol sorting and membrane digestion', Neurochem Res. 2011 Sep;36(9):1594-600 38 T. R. Esch, I. Gadaczek, and T. Bredow, 'Surface structures and thermodynamics of lowindex of rutile, brookite and anatase - A comparative DFT study', Applied Surface Science, vol. 288, pp. 275-287, Jan. 2014.

39 M. Deserno and W. M. Gelbart, 'Adhesion and Wrapping in Colloid-Vesicle Complexes', The Journal of Physical Chemistry B, vol. 106, no. 21, pp. 5543-5552, May 2002.

${ }^{40}$ Martin Müller, Theoretical studies of fluid membrane mechanics, PhD thesis, Johannes Gutenberg-Universitat, Mainz 2007.

41 Claudia Contini, James W. Hindley, Thomas J. Macdonald, Joseph D. Barritt, Oscar Ces, Nick Quirke Size matters: Size Dependency of Gold Nanoparticles Interacting with Model Membranes. Nature Comms: Chemistry (in press)

42 Hana Majaron et al, 'Towards predictive nanotoxicology: From the roundabout of molecular events to chronic inflammation prediction', (submitted for publication)

${ }^{43}$ Nils C. Gauthier, Thomas A. Masters and Michael P. Sheetz, 'Mechanical feedback between membrane tension and dynamics', Trends Cell Biol. 2012; 22(10):527

${ }^{44}$ Drazen Raucher and Michael P. Sheetz, Characteristics of a Membrane Reservoir Buffering Membrane Tension. Biophys. J. 1999; 77(4):1992-2002

${ }^{45}$ C. Dietrich, M. Angelova, and B. Pouligny, 'Adhesion of Latex Spheres to Giant Phospholipid Vesicles: Statics and Dynamics', Journal de Physique II, vol. 7, no. 11, pp. 1651-1682, Nov. 1997, doi: 10.1051/jp2:1997208.

${ }^{46}$ Markus Deserno and William M. Gelbart,' Adhesion and Wrapping in Colloid-Vesicle Complexes', J. Phys. Chem. B 2002, 106, 5543-5552 